\title{
A Substructural Damage Identification Approach for Shear Structure Based on Changes in the First AR Model Coefficient Matrix
}

\author{
Liu Mei, Akira Mita, and Jin Zhou \\ Department of System Design Engineering, Keio University, 3-14-1 Hiyoshi, Kohoku-ku, Yokohama 223-8522, Japan \\ Correspondence should be addressed to Liu Mei; meiliumail@gmail.com
}

Received 15 April 2015; Accepted 1 June 2015

Academic Editor: Elsa de Sá Caetano

Copyright (C) 2015 Liu Mei et al. This is an open access article distributed under the Creative Commons Attribution License, which permits unrestricted use, distribution, and reproduction in any medium, provided the original work is properly cited.

\begin{abstract}
A substructural damage identification approach based on changes in the first AR model coefficient matrix is proposed in this paper to identify structural damage including its location and severity. Firstly, a substructure approach is adopted in the procedure to divide a complete structure into several substructures in order to significantly reduce the number of unknown parameters for each substructure so that damage identification processes can be independently conducted on each substructure. To establish a relation between changes in AR model coefficients and structural damage for each substructure, a theoretical derivation is presented. Thus the accelerations are fed into ARMAX models to determine the AR model coefficients for each substructure under undamaged and various damaged conditions, based on which changes in the first AR model coefficient matrix (CFAR) is obtained and adopted as the damage indicator for the proposed substructure damage identification approach. To better assess the performance of the proposed procedure, a numerical simulation and an experimental verification of the proposed approach are then carried out and the results show that the proposed procedure can successfully locate and quantify the damage in both simulation and laboratory experiment.
\end{abstract}

\section{Introduction}

Research and development of structural health monitoring (SHM) are getting strong attention for evaluating and maintaining structural integrity of an aging building or a suffering structure against natural hazards such as large earthquakes and strong winds [1]. The main parts of the SHM in civil engineering are damage detection and localization, which are essential monitoring zones for structures after major events such as earthquakes.

Damage identification based on vibration data generated by SHM systems has been extensively studied for several decades and the literature on the subject is rather immense. One of the earliest damage indicators studied is the estimated modal property from system identification $[2,3]$, as it is directly related to structural physics according to classical dynamics theory. The basic idea is that modal parameters, such as frequencies, mode shapes, and modal damping, are functions of the physical properties of the structure (mass, stiffness, and damping). Therefore changes in the physical properties will cause changes in the modal properties. Thus they are often referred to as mode-based methods. In 1979, Cawley and Adams [4] proposed frequency shifts as a damage indicator to detect damage in composite materials. Doebling and Farrar [5] considered the changes in the frequencies and mode shapes as the damage indicator of a bridge. A comprehensive summarization of damage identification techniques using changes in natural frequencies was presented by Doebling et al. in [6].

However, the modal parameters are proved to be insensitive to local damage for the fact that they are global properties of the structure while damage is a local phenomenon. Additionally, traditional mode-based methods, especially those that operate in time domain, are usually computationally intensive to implement and physical or finite-element models are necessarily required for those methods. In order to overcome these obstacles, time-series-based methods become another important category within the broader family of 
vibration-based methods for damage identification purposes. Comparing with the traditional mode-based methods, although time-series-based methods are also concerned with numerical modeling, they are more flexible because they are data-based rather than physics-based, which can use various damage features that do not necessarily have an explicit physical meaning [7]. Among those time-series-based methods, the autoregressive (AR) model, the autoregressive with exogenous input (ARX) model or the autoregressive moving average with exogenous input (ARMAX) model is mathematical structures that can be used to formulate various datadriven damage features. By adopting one of the standard algorithms, AR/ARX/ARMAX model parameters can be estimated from input-output datasets very efficiently. According to the specific feature extraction process, the damage features generated from these models can be classified into two categories: model coefficients based and model residual based. There are a lot of model coefficients based techniques that have been investigated during the past decades. Sohn and Farrar [8] proposed statistical pattern recognition methodology in which the recorded dynamic signals were modeled by adopting the AR time-series models and then classified from either undamaged or damaged systems by statistically examining changes in AR coefficients. Nair et al. [9] proposed a sensitive damage feature by only using the first three AR coefficients of the ARMA model. By looking into various changes in coefficients of the vector seasonal autoregressive integrated moving average (ARIMA) model, Omenzetter and Brownjohn [10] proposed a health monitoring algorithm for bridge structure by analyzing the time histories of static strain data. de Lautour and Omenzetter [11] adopted an artificial neural network (ANN) to detect the extent of the damage by feeding the AR coefficients as input features into it. On the other hand, model residual based methods have also been receiving attention and a large amount of related research has been documented and published. Fanning and Carden [12] proposed a statistical process control approach to detect damage by using the mean and variance of the residuals of the AR model to form the statistical process control charts. Mattson and Pandit [13] chose the standard deviation of the residual of the vector AR (VAR) model as the damage-sensitive index.

Though numerous damage identification methods mentioned above are already developed for SHM systems, most of them are not feasible or practical for large-scale civil structures due to the challenges such as high equipment costs, long setup time, difficulties in cabling, and the long computation time. To overcome these problems, some researchers have been using the substructure method for local damage identification of the large-scale structures. Koh et al. [14] are considered to be the first to present the concept of substructure identification. In their approach, the extended Kalman filter (EKF) was used as the numerical tool to identify unknown structural parameters. Yun and Lee [15] applied the sequential prediction error method to estimate unknown parameters of each substructure with noisy measurements. Park et al. [16] proposed structural damage identification methods based on the relative changes in localized flexibility properties, which are obtained either by applying a decomposition procedure to an experimentally determined global flexibility matrix or by processing the output signals of a vibration test in a substructure-by-substructure manner. Tee et al. [17] proposed a substructure identification method considering both first-order and second-order models. Koh and Shankar [18] proposed a substructure identification approach in frequency-domain without the need of interface measurement. Several new substructure methods have been developed in recent years. Xing and Mita [19] proposed a substructure approach to divide a complete structure into several substructures in order to significantly reduce the number of unknown parameters for each substructure so that damage identification processes can be independently conducted on each substructure. Hou et al. [20] proposed and experimentally studied a substructure isolation method which can be applied for local structural health monitoring and damage identification by virtually isolating the substructure from a large and complex global structure into a simple, small, and independent structure. Kuwabara et al. [21] proposed a damage identification method for high-rise buildings which is devised to find the story shear and bending stiffness of a specific story from the floor accelerations just above and below the specific story. Zhang et al. [22] presented a loop substructure identification method to estimate the parameters of any story in a shear structure using the cross power spectral densities (CPSD) of structural responses. Lee and Eun [23] presented a model-based substructuring method in which the damaged substructure is detected by tracing the distribution of the constraint forces at the nodes between the partitioned substructures and the local damage is found by the displacement curvature of the isolated substructure.

However, there are still several obstacles to application of the existing methods in practice. Some of these methods need a large amount of measured data and aim at the identification of the mass, stiffness, and damping matrices of the structure, thus too complicated to require long computation time. And some methods require the construction of structure models, but in many cases such models will be overly complex and possibly even impossible to obtain in reasonable time due to the complex nature of many realistic structural systems. Additionally, some other methods require the application of statistical pattern recognition method, in which training data sets are necessary from both the undamaged and damaged states of a system for the statistical modeling. This kind of methods is always impractical for the fact that it is extremely difficult to obtain the training data sets from various damaged states of the structure especially when it is in constant use. Finally, for some others, noise immunity problems of the methodologies are not verified, which may possibly cause some difficulties in the case of practical application. Thus in this paper, a substructural approach based on changes in the first AR model coefficient matrix has been proposed for local damage identification in shear structures. Firstly, a substructure algorithm is used to divide a complete structure into several substructures, each of which shares a common form of the equation of motion. Then the equation of motion for each substructure is rewritten in terms of ARX model with different inputs and outputs. In what follows, it is derived theoretically that the elements of changes in the first AR 


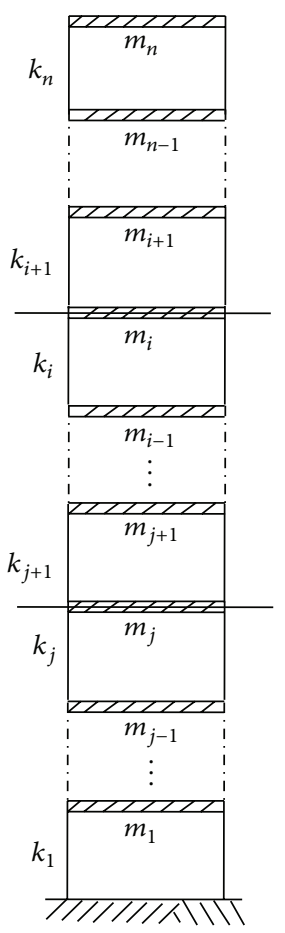

(a) Complete structural system

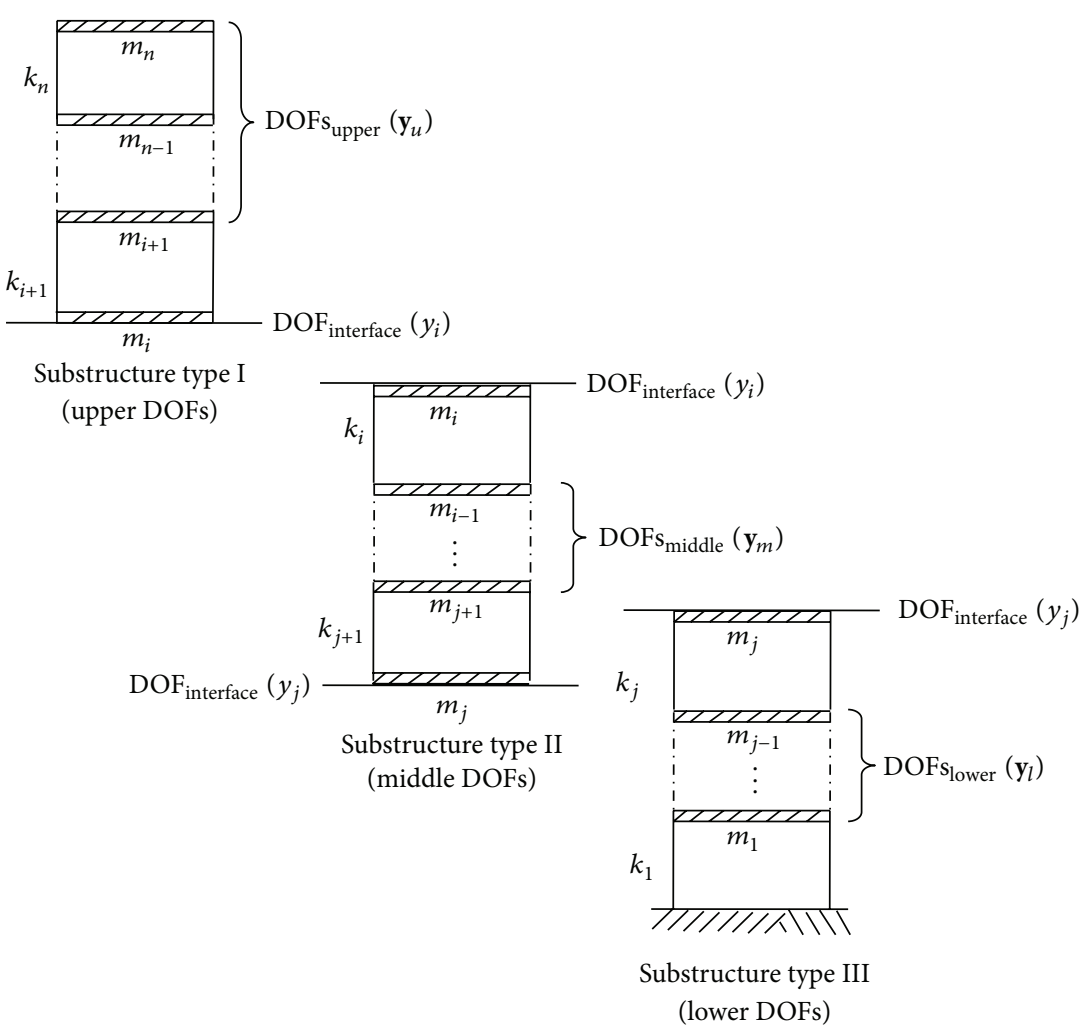

(b) Substructural system

Figure 1: Complete and substructural dynamic system. model coefficient matrix (hereafter will be termed as CFAR) corresponding to the output DOFs adjacent to the damaged location are proportional to the stiffness reduction in the structure, indicating the damage location and severity. Thus the accelerations are fed into autoregressive-moving average with exogenous inputs (ARMAX) models to determine the AR model coefficients for each substructure under undamaged and various damaged conditions, based on which the CFAR is obtained and adopted as the damage indicator for the proposed substructure damage identification approach afterwards. After presenting the proposed approach, a numerical simulation and an experimental verification were conducted and the results were presented to show the feasibility and stability of the proposed methodology. Finally, conclusions and expectations were discussed.

\section{Proposed Method}

To illustrate the concept of substructuring, consider a shear building which is represented by a lumped mass system as shown in Figure 1(a). The dynamic equation of motion for the complete structure is

$$
\mathbf{M} \ddot{\mathbf{y}}(t)+\mathbf{C} \dot{\mathbf{y}}(t)+\mathbf{K y}(t)=-\mathbf{M r} \ddot{z}_{g}(t),
$$

where $\mathbf{M}, \mathbf{C}$, and $\mathbf{K}$ are the mass, damping, and stiffness matrices, respectively, $\mathbf{y}(t), \dot{\mathbf{y}}(t)$, and $\ddot{\mathbf{y}}(t)$ are the dynamic response vectors of displacement, velocity, and acceleration relative to the ground, respectively, $\mathbf{r}$ is an $n \times 1$ unit vector $\left(\mathbf{r}=[1 \cdots 1]^{\mathrm{T}}\right)$, and $\ddot{z}_{g}(t)$ is the ground acceleration.

Without loss of generality, considering the complete structure divided into three parts such as the Substructure I (the upper DOFs), the Substructure II (the middle DOFs) and the Substructure III (the lower DOFs), as shown in Figure 1(b), the dynamic equation of motion (1) can be written in the following partition form:

$$
\begin{aligned}
& {\left[\begin{array}{ccccc}
\mathbf{M}_{u, u} & & & & 0 \\
& m_{i} & & & \\
& & \mathbf{M}_{m, m} & & \\
& & & m_{j} & \\
0 & & & \mathbf{M}_{l, l}
\end{array}\right] \cdot\left\{\begin{array}{c}
\ddot{\mathbf{y}}_{u} \\
\ddot{y}_{i} \\
\ddot{\mathbf{y}}_{m} \\
\ddot{y}_{j} \\
\ddot{\mathbf{y}}_{l}
\end{array}\right\}} \\
& +\left[\begin{array}{cccccc}
\mathbf{C}_{u, u} & \mathbf{C}_{u, i} & & & 0 \\
\mathbf{C}_{i, u} & c_{i+1}+c_{i} & \mathbf{C}_{i, m} & & \\
& \mathbf{C}_{m, i} & \mathbf{C}_{m, m} & \mathbf{C}_{m, j} & \\
& & & \mathbf{C}_{j, m} & c_{j+1}+c_{j} & \mathbf{C}_{j, l} \\
0 & & & \mathbf{C}_{l, j} & \mathbf{C}_{l, l}
\end{array}\right] \cdot\left\{\begin{array}{c}
\dot{\mathbf{y}}_{u} \\
\dot{y}_{i} \\
\dot{\mathbf{y}}_{m} \\
\dot{y}_{j} \\
\dot{\mathbf{y}}_{l}
\end{array}\right\}
\end{aligned}
$$




$$
\begin{aligned}
& +\left[\begin{array}{ccccc}
\mathbf{K}_{u, u} & \mathbf{K}_{u, i} & & & 0 \\
\mathbf{K}_{i, u} & k_{i+1}+k_{i} & \mathbf{K}_{i, m} & & \\
& \mathbf{K}_{m, i} & \mathbf{K}_{m, m} & \mathbf{K}_{m, j} & \\
& & \mathbf{K}_{j, m} & k_{j+1}+k_{j} & \mathbf{K}_{j, l} \\
0 & & & \mathbf{K}_{l, j} & \mathbf{K}_{l, l}
\end{array}\right] \\
& \left\{\begin{array}{l}
\mathbf{y}_{u} \\
y_{i} \\
\mathbf{y}_{m} \\
y_{j} \\
\mathbf{y}_{l}
\end{array}\right\} \\
& =-\left[\begin{array}{ccccc}
\mathbf{M}_{u, u} & & & & 0 \\
& m_{i} & & & \\
& & \mathbf{M}_{m, m} & & \\
& & & m_{j} & \\
0 & & & & \mathbf{M}_{l, l}
\end{array}\right] \cdot\left\{\begin{array}{c}
\mathbf{r}_{u} \\
1 \\
\mathbf{r}_{m} \\
1 \\
\mathbf{r}_{l}
\end{array}\right\} \cdot \ddot{z}_{g},
\end{aligned}
$$

where the subscripts, $u, m$, and $l$, denote inner DOFs of the divided substructures corresponding to the upper, the middle, and the lower parts. And the subscripts, $i$ and $j$, denote interface DOFs of the substructures.

Extracting the row $m$ from (2) and moving the interaction force between the interface and the middle substructure to the right-hand side, the dynamic equation of motion for the middle substructure can be rewritten as follows:

$$
\begin{aligned}
\mathbf{M}_{m, m} \ddot{\mathbf{y}}_{m}(t)+\mathbf{C}_{m, m} \dot{\mathbf{y}}_{m}(t)+\mathbf{K}_{m, m} \mathbf{y}_{m}(t) \\
=-\mathbf{M}_{m, m} \mathbf{r}_{m} \ddot{z}_{g}(t)-\mathbf{C}_{m, i} \dot{y}_{i}(t)-\mathbf{C}_{m, j} \dot{y}_{j}(t) \\
\quad-\mathbf{K}_{m, i} y_{i}(t)-\mathbf{K}_{m, j} y_{j}(t) .
\end{aligned}
$$

From the terms in the right-hand side, it can be found that the interaction effects at the interface ends can be treated as "input" so the new input to the substructure includes two parts: the system input and the interface force input. And the computation of the interface force input requires the measurements of velocities and displacements at the interface DOFs. However, in reality it is usually much easier and preferred to measure accelerations (by accelerometers) than velocities and displacements. Therefore, for practicability, to eliminate the requirement of velocities and displacements, the idea of "quasistatic displacement" vector proposed by Koh et al. [24] is adopted. The displacement relative to the ground DOF is split into a quasistatic displacement term, $\mathbf{y}_{m}^{s}(t)$, and a displacement term relative to the interface ends, $\mathbf{y}_{m}^{r}(t)$; that is,

$$
\mathbf{y}_{m}(t)=\mathbf{y}_{m}^{s}(t)+\mathbf{y}_{m}^{r}(t)
$$

The former term can be obtained by solving (3) while ignoring all time-derivative terms and the dynamic excitation:

$$
\mathbf{y}_{m}^{s}(t)=-\mathbf{K}_{m, m}^{-1} \mathbf{K}_{m, i} y_{i}(t)-\mathbf{K}_{m, m}^{-1} \mathbf{K}_{m, j} y_{j}(t) .
$$

Substituting (4) and (5) into (3) leads to the dynamic equation of motion for the middle substructure:

$$
\begin{aligned}
\mathbf{M}_{m, m} \ddot{\mathbf{y}}_{m}^{r}(t)+\mathbf{C}_{m, m} \dot{\mathbf{y}}_{m}^{r}(t)+\mathbf{K}_{m, m} \mathbf{y}_{m}^{r}(t) \\
=-\mathbf{M}_{m, m} \mathbf{r}_{m} \ddot{z}_{g}(t)+\mathbf{M}_{m, m} \mathbf{K}_{m, m}^{-1} \mathbf{K}_{m, i} \ddot{y}_{i}(t) \\
+\mathbf{M}_{m, m} \mathbf{K}_{m, m}^{-1} \mathbf{K}_{m, j} \ddot{y}_{j}(t) \\
-\left(\mathbf{C}_{m, i}-\mathbf{C}_{m, m} \mathbf{K}_{m, m}^{-1} \mathbf{K}_{m, i}\right) \dot{y}_{i}(t) \\
-\left(\mathbf{C}_{m, j}-\mathbf{C}_{m, m} \mathbf{K}_{m, m}^{-1} \mathbf{K}_{m, j}\right) \dot{y}_{j}(t) .
\end{aligned}
$$

Similarly for the upper and lower substructures, the dynamic equation of motion can be obtained as follows:

$$
\begin{aligned}
\mathbf{M}_{u, u} \ddot{\mathbf{y}}_{u}^{r}(t)+\mathbf{C}_{u, u} \dot{\mathbf{y}}_{u}^{r}(t)+\mathbf{K}_{u, u} \mathbf{y}_{u}^{r}(t) \\
=-\mathbf{M}_{u, u} \mathbf{r}_{u} \ddot{z}_{g}(t)+\mathbf{M}_{u, u} \mathbf{K}_{u, u}^{-1} \mathbf{K}_{u, i} \ddot{y}_{i}(t) \\
-\left(\mathbf{C}_{u, i}-\mathbf{C}_{u, u} \mathbf{K}_{u, u}^{-1} \mathbf{K}_{u, i}\right) \dot{y}_{i}(t), \\
\mathbf{M}_{l, l} \ddot{\mathbf{y}}_{l}^{r}(t)+\mathbf{C}_{l, l} \dot{\mathbf{y}}_{l}^{r}(t)+\mathbf{K}_{l, l} \mathbf{y}_{l}^{r}(t) \\
=-\mathbf{M}_{l, l} \mathbf{r}_{l} \ddot{z}_{g}(t)+\mathbf{M}_{l, l} \mathbf{K}_{l, l}^{-1} \mathbf{K}_{l, j} \ddot{y}_{j}(t) \\
-\left(\mathbf{C}_{l, j}-\mathbf{C}_{l, l} \mathbf{K}_{l, l}^{-1} \mathbf{K}_{l, j}\right) \dot{y}_{j}(t),
\end{aligned}
$$

where the dynamic response terms with the superscript $r$, $\mathbf{y}^{r}, \dot{\mathbf{y}}^{r}$, and $\ddot{\mathbf{y}}^{r}$, denote the dynamic responses relative to the interface ends. The subscripts, $m, u$, and $l$, denote the DOFs corresponding to the middle, the upper, and the lower substructures.

Since damping force is usually much smaller compared to inertia force in typical civil engineering structures, the velocity-dependent components in the interface motion forces are assumed to be negligible [24]. Hence, (6) and (7) can be simplified as follows:

$$
\begin{aligned}
\mathbf{M}_{m, m} \ddot{\mathbf{y}}_{m}^{r}(t)+\mathbf{C}_{m, m} \dot{\mathbf{y}}_{m}^{r}(t)+\mathbf{K}_{m, m} \mathbf{y}_{m}^{r}(t) & =\mathbf{L}_{m} \mathbf{u}_{m}(t), \\
\mathbf{M}_{u, u} \ddot{\mathbf{y}}_{u}^{r}(t)+\mathbf{C}_{u, u} \dot{\mathbf{y}}_{u}^{r}(t)+\mathbf{K}_{u, u} \mathbf{y}_{u}^{r}(t) & =\mathbf{L}_{u} \mathbf{u}_{u}(t), \\
\mathbf{M}_{l, l} \ddot{\mathbf{y}}_{l}^{r}(t)+\mathbf{C}_{l, l} \dot{\mathbf{y}}_{l}^{r}(t)+\mathbf{K}_{l, l} \mathbf{y}_{l}^{r}(t) & =\mathbf{L}_{l} \mathbf{u}_{l}(t),
\end{aligned}
$$

where $\mathbf{u}_{m}(t), \mathbf{u}_{u}(t)$, and $\mathbf{u}_{l}(t)$ are the input vectors for the middle, upper, and lower substructures, respectively, and $\mathbf{u}_{m}(t)=\left[\begin{array}{lll}\ddot{z}_{g}(t) & \ddot{y}_{i}(t) & \ddot{y}_{j}(t)\end{array}\right]^{\mathrm{T}}, \mathbf{u}_{u}(t)=\left[\begin{array}{ll}\ddot{z}_{g}(t) & \ddot{y}_{i}(t)\end{array}\right]^{\mathrm{T}}, \mathbf{u}_{l}(t)=$ $\left[\begin{array}{ll}\ddot{z}_{g}(t) & \ddot{y}_{i}(t)\end{array}\right]^{\mathrm{T}}$, and we can see that there are 3 inputs for the 
middle substructure and 2 inputs for the upper and lower substructures and $\mathbf{L}_{m}, \mathbf{L}_{\mathcal{u}}$, and $\mathbf{L}_{l}$ are the input coefficient matrices for the middle, upper, and lower substructures, respectively, and $\mathbf{L}_{m}=\left[\begin{array}{lll}-\mathbf{M}_{m, m} \mathbf{r}_{m} & \mathbf{M}_{m, m} \mathbf{K}_{m, m}^{-1} \mathbf{K}_{m, i} & \mathbf{M}_{m, m} \mathbf{K}_{m, m}^{-1} \mathbf{K}_{m, j}\end{array}\right], \mathbf{L}_{u}=$ $\left[\begin{array}{lll}-\mathbf{M}_{u, u} \mathbf{r}_{u} & \mathbf{M}_{u, u} \mathbf{K}_{u, u}^{-1} \mathbf{K}_{u, i}\end{array}\right]$, and $\mathbf{L}_{l}=\left[\begin{array}{ll}-\mathbf{M}_{l, l} \mathbf{r}_{l} & \mathbf{M}_{l, l} \mathbf{K}_{l, l}^{-1} \mathbf{K}_{l, j}\end{array}\right]$.

Equations (8) show that the equation of motion for the middle, upper, and lower substructures takes the same form as follows:

$$
\mathbf{M}_{s, s} \ddot{\mathbf{y}}_{s}^{r}(t)+\mathbf{C}_{s, s} \dot{\mathbf{y}}_{s}^{r}(t)+\mathbf{K}_{s, s} \mathbf{y}_{s}^{r}(t)=\mathbf{L}_{s} \mathbf{u}_{s}(t)
$$

where the subscript $s$ can be substituted with $m, u$, and $l$, denoting all the three types of substructures.

Equation (9) can be rewritten as

$$
\ddot{\mathbf{y}}_{s}^{r}(t)+\widetilde{\mathbf{C}}_{s, s} \dot{\mathbf{y}}_{s}^{r}(t)+\widetilde{\mathbf{K}}_{s, s} \mathbf{y}_{s}^{r}(t)=\widetilde{\mathbf{L}}_{s} \mathbf{u}_{s}(t)
$$

where $\widetilde{\mathbf{C}}_{s, s}=\mathbf{M}_{s, s}^{-1} \mathbf{C}_{s, s}, \widetilde{\mathbf{K}}_{s, s}=\mathbf{M}_{s, s}^{-1} \mathbf{K}_{s, s}$, and $\widetilde{\mathbf{L}}_{s}=\mathbf{M}_{s, s}^{-1} \mathbf{L}_{s}$.

The knowledge of dynamics tells us that the mass normalized stiffness $\widetilde{\mathbf{K}}_{s, s}$ can be expressed as $\boldsymbol{\Phi}_{s} \boldsymbol{\Lambda}_{s} \boldsymbol{\Phi}_{s}^{\mathrm{T}}$, where $\boldsymbol{\Phi}_{s}$ and $\Lambda_{s}$ are the mass normalized mode shape and natural (circular) frequency matrices for the corresponding substructure, respectively. Lu and Gao [25] prove that (10) can be written in terms of ARX model as follows:

$$
\widehat{\mathbf{y}}_{s}^{r}(t)=\sum_{i=1}^{2} \mathbf{A}_{i} \mathbf{y}_{s}^{r}(t-i)+\sum_{j=1}^{3} \mathbf{B}_{j} \mathbf{u}_{s}(t-j)
$$

where $\widehat{\mathbf{y}}_{s}^{r}(t)$ is the predicted signal, $\mathbf{A}_{1}=2 \Phi_{s} \cos \left(\Lambda_{s}^{1 / 2} \Delta t\right) \Phi_{s}^{\mathrm{T}}$, $\mathbf{A}_{2}=-\mathbf{I}, \mathbf{B}_{1}=\widetilde{\mathbf{L}}_{s}, \mathbf{B}_{2}=-\boldsymbol{\Phi}_{s}\left[\mathbf{I}+\cos \left(\Lambda_{s}^{1 / 2} \Delta t\right)\right] \boldsymbol{\Phi}_{s}^{\mathrm{T}} \widetilde{\mathbf{L}}_{s}$, and $\mathbf{B}_{3}=$ $\boldsymbol{\Phi}_{s} \cos \left(\Lambda_{s}^{1 / 2} \Delta t\right) \boldsymbol{\Phi}_{s}^{\mathrm{T}} \tilde{\mathbf{L}}_{s}$ with $\Delta t$ being the sampling period of the dynamic response measurement and I being the identity matrix.

Obviously, the ARX model coefficients are governed by the structural parameters (i.e., mass, stiffness, etc.) as shown by $\mathrm{Lu}$ and Gao [25]. Hence any sorts of deterioration in structural properties will lead to a change in the ARX model coefficients. Now, let us consider a shear structure which has undergone some damage. Thus, the first AR model coefficient matrices corresponding to the undamaged and damaged states can be termed as $\mathbf{A}_{1}^{u}$ and $\mathbf{A}_{1}^{d}$, respectively.

We have $\widetilde{\mathbf{K}}_{s, s}=\Phi_{s} \Lambda_{s} \Phi_{s}^{T}=\Phi_{s} \Lambda_{s}^{1 / 2} \Lambda_{s}^{1 / 2} \Phi_{s}^{T}=$ $\boldsymbol{\Phi}_{s} \Lambda_{s}^{1 / 2} \boldsymbol{\Phi}_{s}^{\mathrm{T}} \boldsymbol{\Phi}_{s} \Lambda_{s}^{1 / 2} \boldsymbol{\Phi}_{s}^{\mathrm{T}}=\left(\boldsymbol{\Phi}_{s} \Lambda_{s}^{1 / 2} \boldsymbol{\Phi}_{s}^{\mathrm{T}}\right)^{2}$. It follows that $\boldsymbol{\Phi}_{s} \Lambda_{s}^{1 / 2} \boldsymbol{\Phi}_{s}^{\mathrm{T}}=\widetilde{\mathbf{K}}_{s, s}^{1 / 2}$. Now the first AR model coefficient matrix can be expressed as follows:

$$
\begin{aligned}
\mathbf{A}_{1}= & 2 \Phi_{s} \cos \left(\Lambda_{s}^{1 / 2} \Delta t\right) \Phi_{s}^{\mathrm{T}}=2 \cos \left(\widetilde{\mathbf{K}}_{s, s}^{1 / 2} \Delta t\right) \\
= & 2 \mathbf{I}-\frac{2}{2 !} \widetilde{\mathbf{K}}_{s, s} \Delta t^{2}+\frac{2}{4 !} \widetilde{\mathbf{K}}_{s, s}^{2} \Delta t^{4}-\frac{2}{6 !} \widetilde{\mathbf{K}}_{s, s}^{3} \Delta t^{6} \\
& +\cdots \infty .
\end{aligned}
$$

Thus the change in the first AR model coefficient matrix from the undamaged state to the damaged state can be expressed as

$$
\begin{aligned}
\Delta \mathbf{A}_{1}= & \mathbf{A}_{1}^{u}-\mathbf{A}_{1}^{d} \\
= & -\frac{2}{2 !}\left(\widetilde{\mathbf{K}}_{s, s}^{u}-\widetilde{\mathbf{K}}_{s, s}^{d}\right) \Delta t^{2} \\
& +\frac{2}{4 !}\left[\left(\widetilde{\mathbf{K}}_{s, s}^{u}\right)^{2}-\left(\widetilde{\mathbf{K}}_{s, s}^{d}\right)^{2}\right] \Delta t^{4} \\
& -\frac{2}{6 !}\left[\left(\widetilde{\mathbf{K}}_{s, s}^{u}\right)^{3}-\left(\widetilde{\mathbf{K}}_{s, s}^{d}\right)^{3}\right] \Delta t^{6}+\cdots \infty \\
= & \sum_{p=1}^{\infty}(-1)^{p} \frac{2}{(2 p) !}\left[\left(\widetilde{\mathbf{K}}_{s, s}^{u}\right)^{p}-\left(\widetilde{\mathbf{K}}_{s, s}^{d}\right)^{p}\right] \Delta t^{2 p} .
\end{aligned}
$$

Now, assuming that the damage occurred on the $f$ th story of the shear structure building, it can be simulated as a stiffness reduction of that story by an amount of $\delta k_{f}$ from its original stiffness, $k_{f}$. Considering the damaged story locating in three different substructures, the modified stiffness matrix of the damaged substructure takes the forms in three different cases as follows:

(1) $i+1 \leqslant f \leqslant n$, in case of damaged story in upper substructure

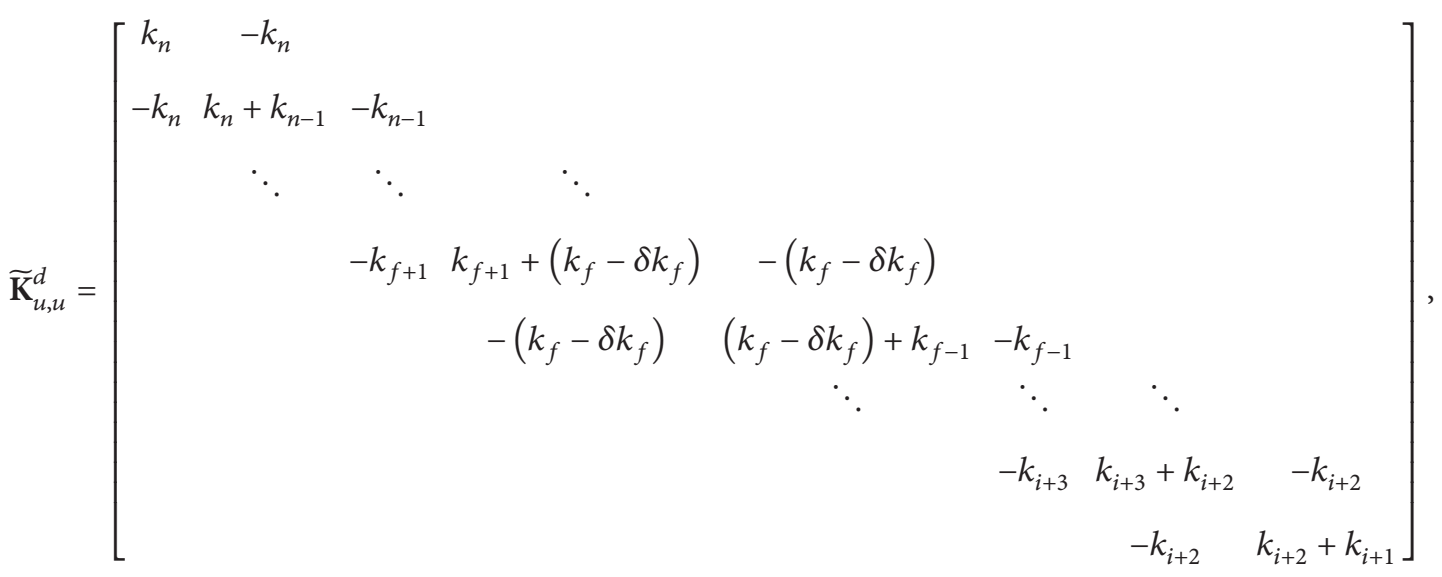


(2) $j+1 \leqslant f \leqslant i$, in case of damaged story in middle substructure

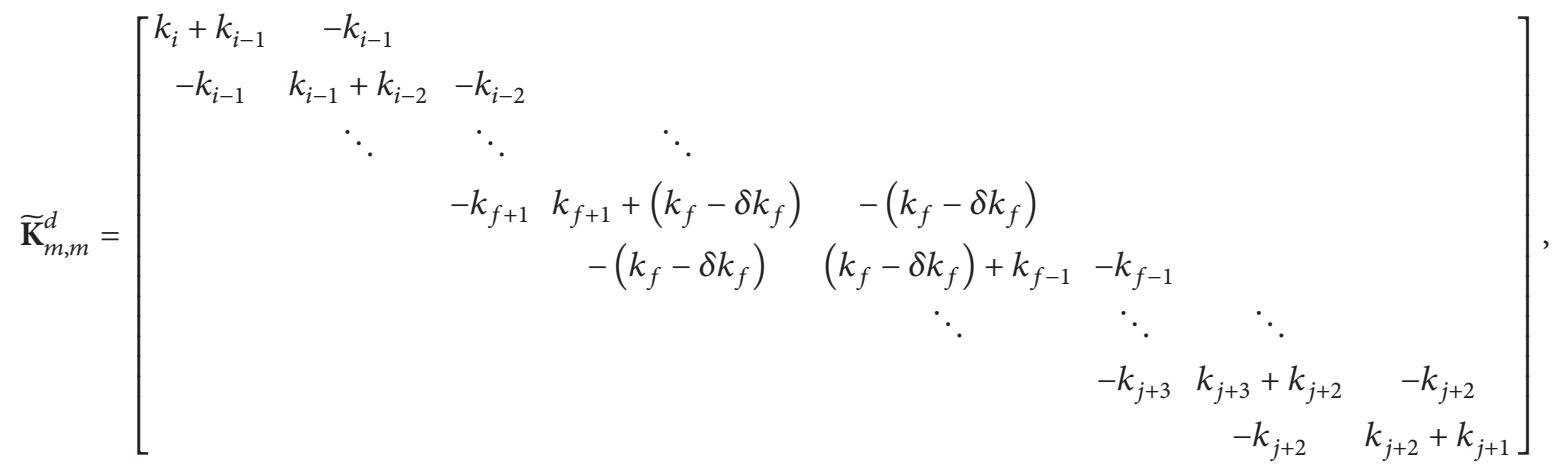

(3) $1 \leqslant f \leqslant j$, in case of damaged story in lower substructure

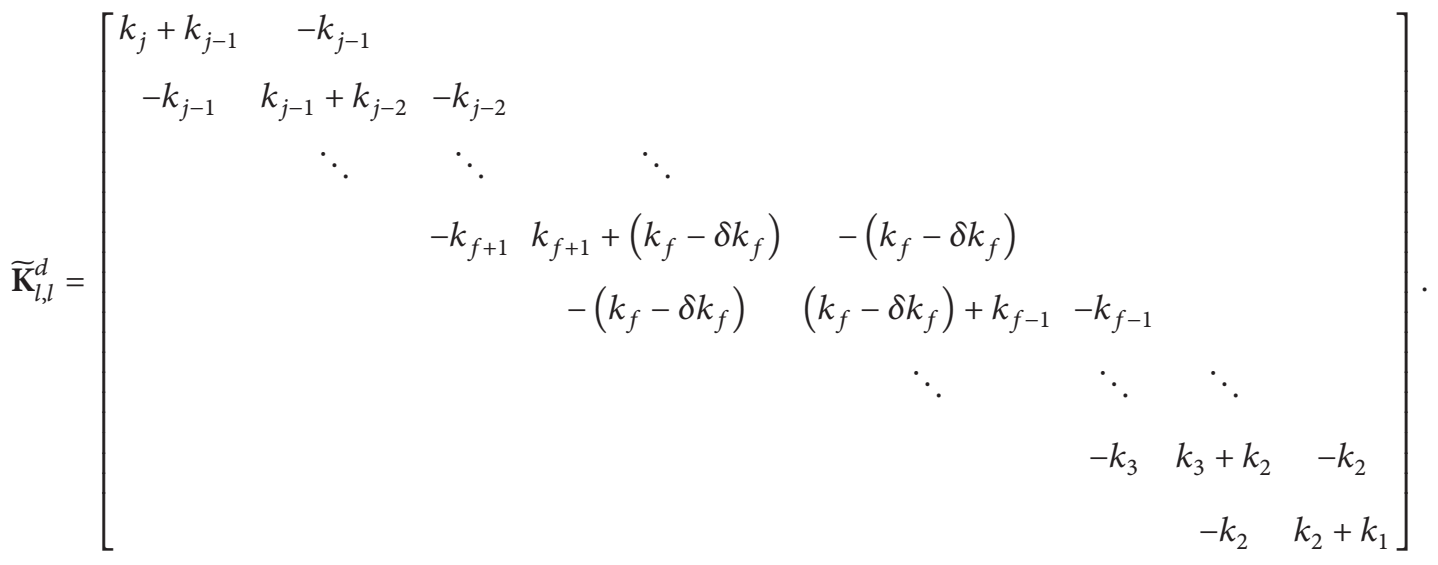

In a uniform way, the stiffness matrix of the damaged substructure in all three cases above can be expressed as $\widetilde{\mathbf{K}}_{s, s}^{d}=$ $\widetilde{\mathbf{K}}_{s, s}^{u}+\Delta \mathbf{K}_{f}$ with $\Delta \mathbf{K}_{f}=\mathbf{I}_{f} \delta k_{f}$, and $\mathbf{I}_{f}$ can be written in matrix form in three different cases as follows:

(1) in case of damaged story nonadjacent to the interface end

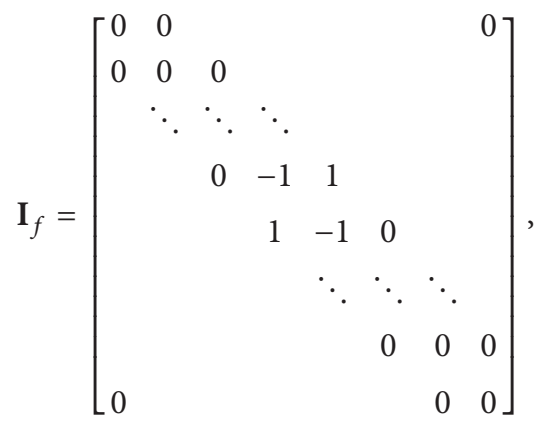

(2) in case of damaged story adjacent to the top interface end

$$
\mathbf{I}_{f}=\left[\begin{array}{cccccccc}
-1 & 0 & & & & & & 0 \\
0 & 0 & 0 & & & & & \\
& \ddots & \ddots & \ddots & & & & \\
& & 0 & 0 & 0 & & & \\
& & & 0 & 0 & 0 & & \\
& & & & \ddots & \ddots & \ddots & \\
& & & & & 0 & 0 & 0 \\
& & & & & 0 & & \\
0 & & & & & & 0 & 0
\end{array}\right],
$$


(3) in case of damaged story adjacent to the bottom interface end

$$
\mathbf{I}_{f}=\left[\begin{array}{cccccccc}
0 & 0 & & & & & & 0 \\
0 & 0 & 0 & & & & & \\
& \ddots & \ddots & \ddots & & & & \\
& & 0 & 0 & 0 & & & \\
& & & 0 & 0 & 0 & & \\
& & & & \ddots & \ddots & \ddots & \\
& & & & & 0 & 0 & 0 \\
0 & & & & & & 0 & -1
\end{array}\right] .
$$

By representing $\widetilde{\mathbf{K}}_{s, s}^{d}$ with $\widetilde{\mathbf{K}}_{s, s}^{u}, \mathbf{I}_{f}$, and $\delta k_{f}$, the term $\left[\left(\widetilde{\mathbf{K}}_{s, s}^{d}\right)^{p}-\left(\widetilde{\mathbf{K}}_{s, s}^{u}\right)^{p}\right]$ of (13) can be rewritten as follows:

$$
\begin{aligned}
& \left(\widetilde{\mathbf{K}}_{s, s}^{d}\right)^{p}-\left(\widetilde{\mathbf{K}}_{s, s}^{u}\right)^{p} \\
& \quad=\left(\widetilde{\mathbf{K}}_{s, s}^{u}+\mathbf{I}_{f} \delta k_{f}\right)^{p}-\left(\widetilde{\mathbf{K}}_{s, s}^{u}\right)^{p} \\
& \quad=\sum_{q=1}^{\infty} C_{q}^{p}\left[\left(\widetilde{\mathbf{K}}_{s, s}^{u}\right)^{-1} \mathbf{I}_{f}\right]^{q}\left(\widetilde{\mathbf{K}}_{s, s}^{u}\right)^{q}\left(\delta k_{f}\right)^{q}
\end{aligned}
$$

where $C_{q}^{p}$ denotes the usual binomial coefficient and $C_{q}^{p}=$ $p ! / q !(p-q) !$.

Substituting (20) into (13) leads to

$$
\begin{aligned}
\Delta \mathbf{A}_{1}= & -\sum_{p=1}^{\infty}(-1)^{p} \frac{2}{(2 p) !} \\
& \cdot \sum_{q=1}^{\infty} C_{q}^{p}\left[\left(\widetilde{\mathbf{K}}_{s, s}^{u}\right)^{-1} \mathbf{I}_{f}\right]^{q}\left(\widetilde{\mathbf{K}}_{s, s}^{u}\right)^{q}\left(\delta k_{f}\right)^{q} \Delta t^{2 p}
\end{aligned}
$$

For small value of story stiffness degradation on the $f$ th story $\left(\delta k_{f}\right)$, the higher order terms of $\delta k_{f}$ can be neglected; that is, only the term with $q=1$ is retained leading (21) into the following form:

$$
\Delta \mathbf{A}_{1}=-\sum_{p=1}^{\infty}(-1)^{p} \frac{2}{(2 p) !} p\left(\widetilde{\mathbf{K}}_{s, s}^{u}\right)^{-1} \mathbf{I}_{f} \widetilde{\mathbf{K}}_{s, s}^{u} \delta k_{f} \Delta t^{2 p}
$$

Similarly, for small time step, $\Delta t^{2 p}$ turns to be negligible as $p$ increases. Thus, only the term with $p=1$ is kept and one can write

$$
\Delta \mathbf{A}_{1}=\left(\widetilde{\mathbf{K}}_{s, s}^{u}\right)^{-1} \mathbf{I}_{f} \widetilde{\mathbf{K}}_{s, s}^{u} \Delta t^{2} \delta k_{f}
$$

Utilizing the expressions of $\mathbf{I}_{f}$ in (17), (18), and (19), the changes in the first AR model coefficient matrix from the undamaged state to the damaged state can be expressed in matrix form in three different cases as follows:

(1) in case of damaged story nonadjacent to the interface end

$$
\Delta \mathbf{A}_{1}=\left[\begin{array}{cccccccc}
0 & 0 & & & & & & 0 \\
0 & 0 & 0 & & & & \\
& \ddots & \ddots & \ddots & & & \\
& & 0 & -\Delta t^{2} \delta k_{f} & \Delta t^{2} \delta k_{f} & & & \\
& & & \Delta t^{2} \delta k_{f} & -\Delta t^{2} \delta k_{f} & 0 & & \\
& & & \ddots & \ddots & \ddots & \\
& & & & & 0 & 0 & 0 \\
0 & & & & & & 0 & 0
\end{array}\right],
$$

(2) in case of damaged story adjacent to the top interface end

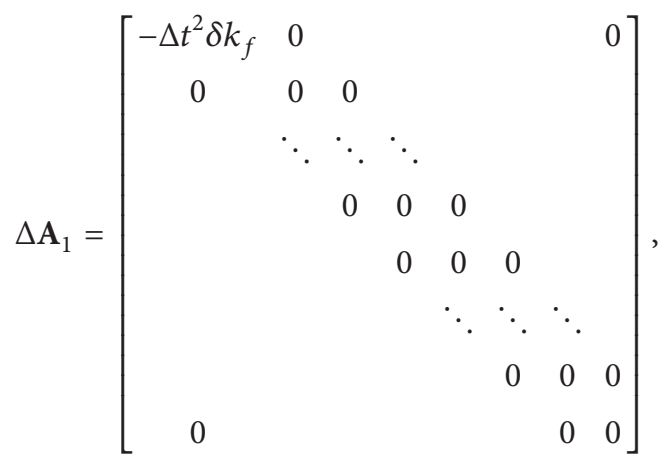

(3) in case of damaged story adjacent to the bottom interface end

$$
\Delta \mathbf{A}_{1}=\left[\begin{array}{cccccccc}
0 & 0 & & & & & & 0 \\
0 & 0 & 0 & & & & & \\
& \ddots & \ddots & \ddots & & & & \\
& & 0 & 0 & 0 & & & \\
& & & 0 & 0 & 0 & & \\
& & & & \ddots & \ddots & \ddots & \\
& & & & & 0 & 0 & 0 \\
0 & & & & & & 0 & -\Delta t^{2} \delta k_{f}
\end{array}\right]
$$

Equations (24), (25), and (26) show that the absolute value of $\Delta \mathbf{A}_{1}$ will be a null matrix with four nonzero terms at element locations corresponding to the output DOFs ( $f$ $1, f-1),(f-1, f),(f, f-1)$, and $(f, f)$ in case of damaged story nonadjacent to the interface end; with one nonzero term at element location corresponding to the output DOF $(f-1, f-1)$ in case of damaged story adjacent to the top interface end; and with one nonzero term at element location corresponding to the output $\operatorname{DOF}(f, f)$ in case of damaged story adjacent to the bottom interface end. This information depicts the damage location on the $f$ th story. Additionally, 


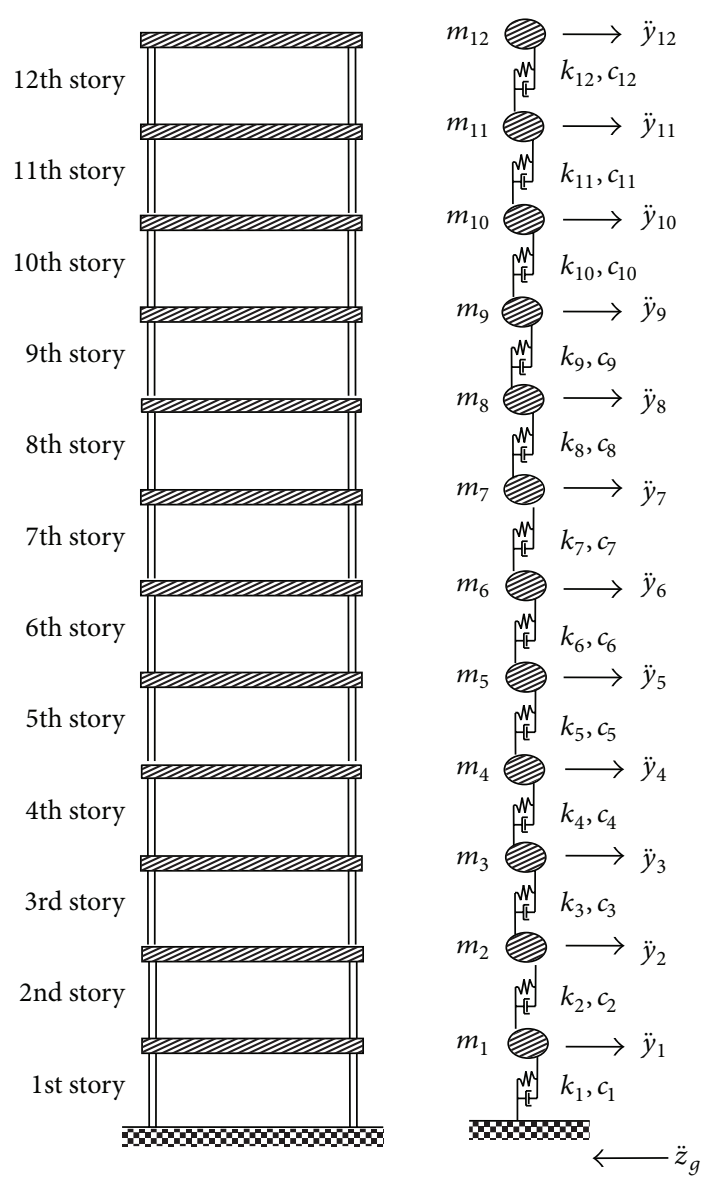

FIGURE 2: 12-story shear building.

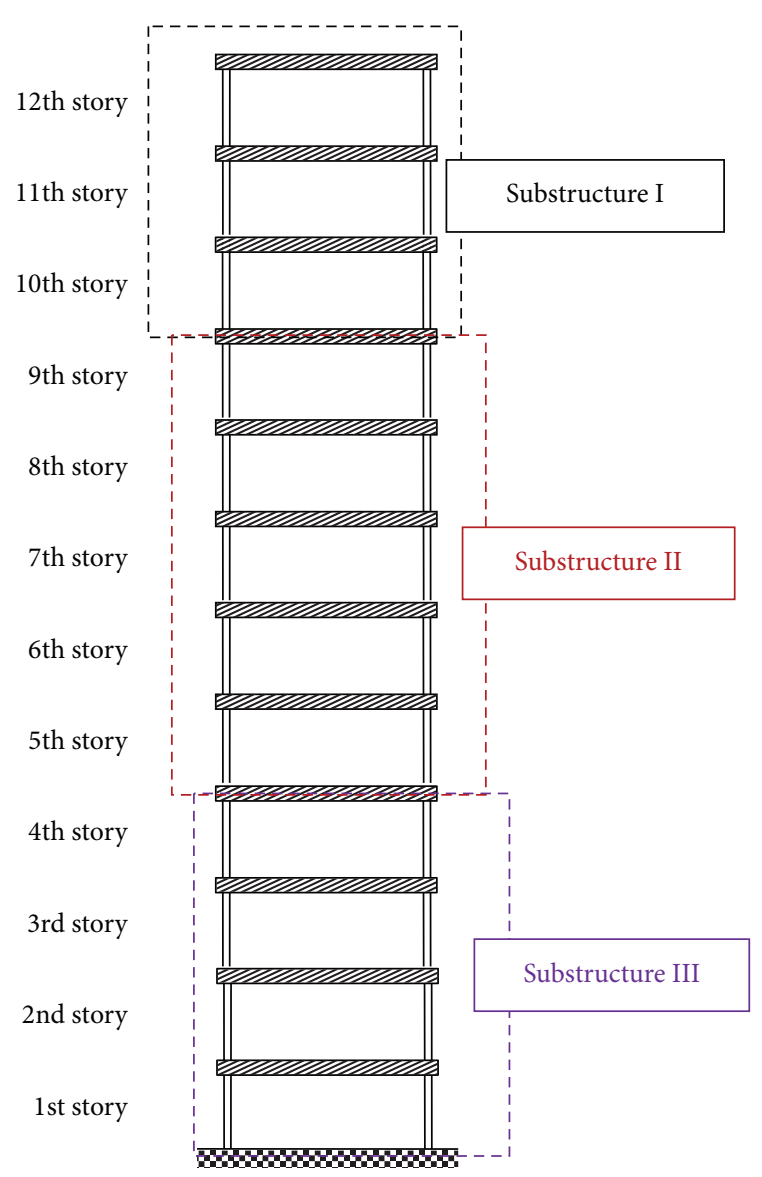

FIGURE 3: Structure division for 12-story shear building. the value of the nonzero term of $\Delta \mathbf{A}_{1}$ is directly proportional to the story stiffness degradation. Thus, the damage can be localized and quantified by identifying these four (or one) high-valued elements in the matrix, $\Delta \mathbf{A}_{1}$. Therefore, the changes in the first AR model coefficient matrix (CFAR) can be used as a damage indicator for the proposed substructural damage identification approach.

\section{Verification by Simulation}

A simulation of a 12-story shear building model was performed to show the feasibility of the proposed scheme for damage identification. The building is simplified into a 12DOF lumped mass structural system as shown in Figure 2.

The mass of every floor and the lateral stiffness were assumed to be $100 \mathrm{~kg}$ and $1 \mathrm{MN} / \mathrm{m}$, respectively. 3\% was chosen as the damping ratio of all modes. The first three undamaged natural frequencies of the structure were calculated as $1.9987 \mathrm{~Hz}, 5.9645 \mathrm{~Hz}$, and $9.8363 \mathrm{~Hz}$ for the 1st mode, 2nd mode, and 3rd mode, respectively. The 12 -DOF system was simulated to be subjected to white noise excitation, and $5 \%$ noise was added to the acceleration responses of the structure. The acceleration responses at different DOFs were recorded for 50 seconds with a sampling frequency of $200 \mathrm{~Hz}$. The story stiffness reduction was regarded to be damage to the structure. Twelve damage cases (damage in each story from the 1st story through the 12th story) with five different damage severities (10\%, 20\%, 30\%, 40\%, and 50\% lateral stiffness reduction) were studied. Hence, there were 60 different damage scenarios in total.

Firstly, according to the substructure division method described in Section 2, the whole structure was divided into 3 substructures, as shown in Figure 3.

Considering strong flexibility for handling the disturbance modeling, the autoregressive moving average with exogenous inputs (ARMAX) model was adopted to model each substructure and further obtain the AR coefficient matrix for the undamaged and unknown states. According to (11) and the expression of the input vector $\mathbf{u}_{u}(t)$, Substructure I can be modeled as a 2-input 3-output ARMAX model. The ground acceleration $\left(\ddot{z}_{q}\right)$ and the acceleration of the 9th DOF relative to the ground $\left(\ddot{y}_{9}\right)$ were used as the inputs, and the relative acceleration of the 10 th, 11 th, and 12 th DOF to the 9th DOF $\left(\ddot{y}_{10}^{r}, \ddot{y}_{11}^{r}\right.$, and $\left.\ddot{y}_{12}^{r}\right)$ was used as the outputs to construct the ARMAX model.

Similarly, Substructure II can be modeled as a 3-input 4output ARMAX model. The ground acceleration $\left(\ddot{z}_{g}\right)$ and the acceleration of the 4 th and 9 th DOF relative to the ground $\left(\ddot{y}_{4}\right.$ and $\ddot{y}_{9}$ ) were used as the inputs, and the relative acceleration of the 5th, 6th, 7th, and 8th DOF to the 4 th DOF $\left(\ddot{y}_{5}^{r}, \ddot{y}_{6}^{r}\right.$, $\ddot{y}_{7}^{r}$, and $\ddot{y}_{8}^{r}$ ) was used as the outputs. Substructure III can be 


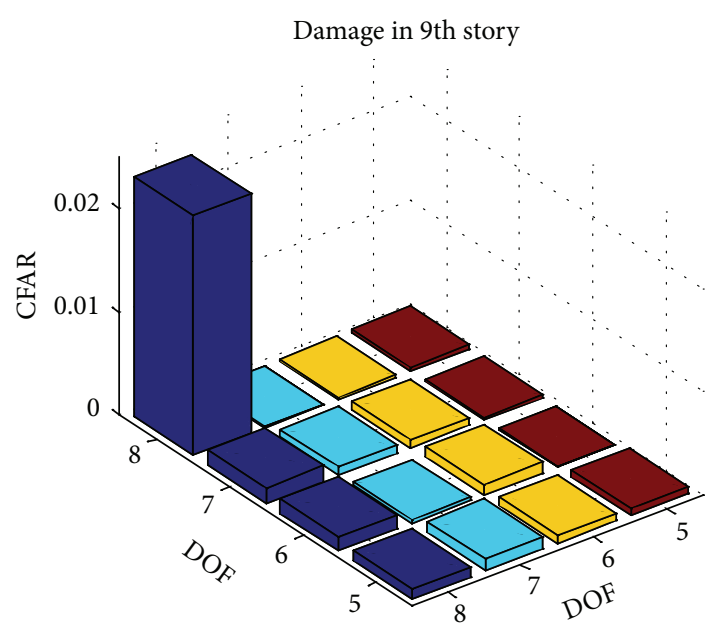

Damage in 7th story

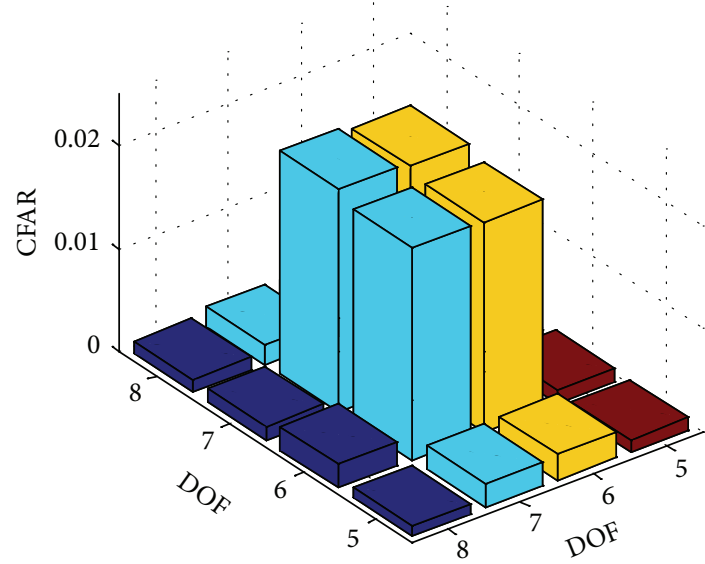

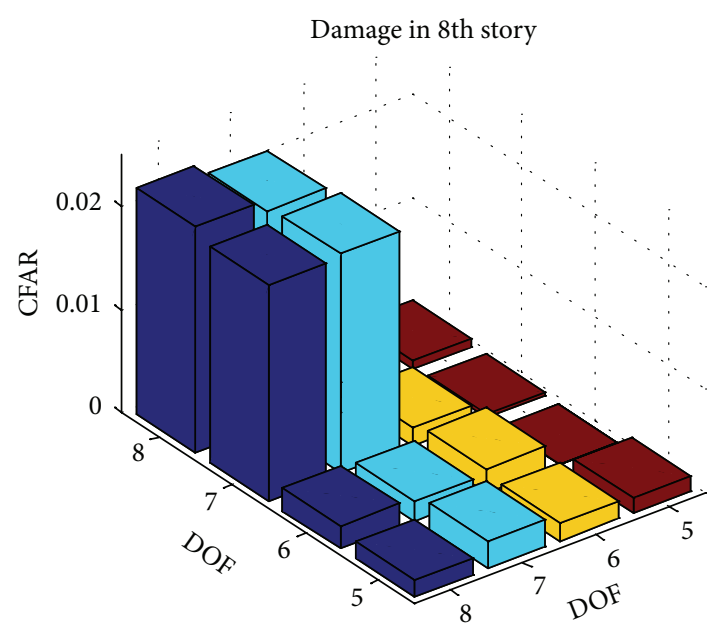

Damage in 6th story

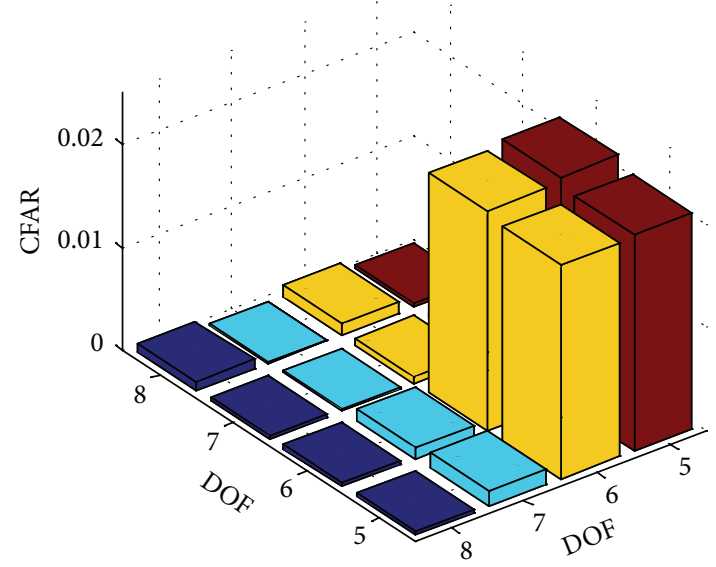

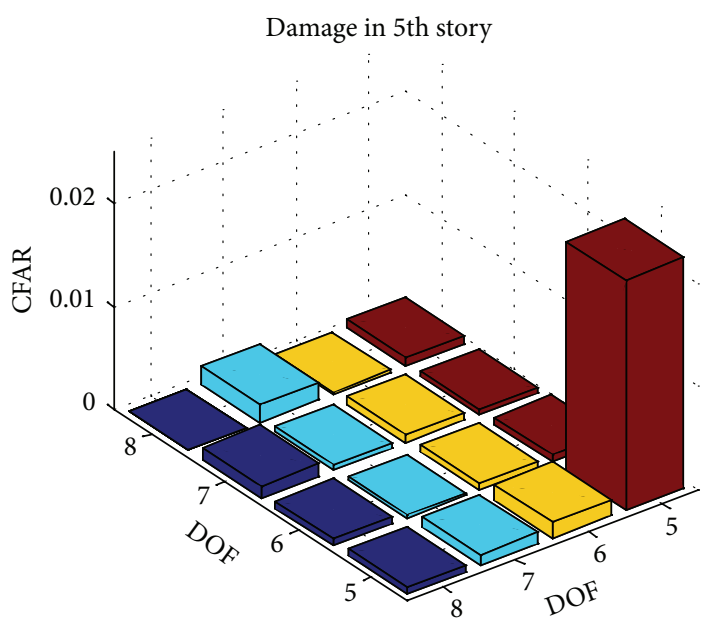

FIGURE 4: Changes in the first AR model coefficient matrix for Substructure II (10\% damage).

modeled as a 2-input 3-output ARMAX model. The ground acceleration $\left(\ddot{z}_{q}\right)$, and the acceleration of the 4 th DOF relative to the ground $\left(\ddot{y}_{4}\right)$ were used as the inputs, and the relative acceleration of the 1st, $2 \mathrm{nd}$, and 3rd DOF to the 4 th DOF $\left(\ddot{y}_{1}^{r}\right.$, $\ddot{y}_{2}^{r}$, and $\ddot{y}_{3}^{r}$ ) was used as the outputs.

The Burg's (maximum entropy) method (BM) was used to identify the ARMAX model coefficients from input-output data sets for each substructure and each damage scenario. The changes in the first AR model coefficient matrix (CFAR) from the undamaged state to the damaged states were estimated. Figure 4 shows a three-dimensional bar plot of the CFAR against output DOFs for Substructure II in the case of $10 \%$ damage occurring in 9th story, 8th story, 7th story, 6th story, and 5th story, respectively. The absolute values of CFAR are 
TABLE 1: Changes in the first AR model coefficient matrix for Substructure I (10\% damage).

\begin{tabular}{lccc}
\hline DOF & 12th story & 11th story & 10th story \\
\hline 12th story & $\mathbf{0 . 0 2 2 1}$ & Damage in 12th story \\
11th story & $\mathbf{0 . 0 2 2 2}$ & $\mathbf{0 . 0 2 1 1}$ & 0.0011 \\
10th story & 0.0008 & 0.0007 & 0.0004 \\
\hline \multicolumn{3}{c}{ Damage in 11th story } \\
12th story & 0.0012 & 0.0023 & 0.0001 \\
11th story & 0.0003 & $\mathbf{0 . 0 2 2 5}$ & $\mathbf{0 . 0 2 2 3}$ \\
10th story & 0.0017 & $\mathbf{0 . 0 2 1 7}$ & $\mathbf{0 . 0 2 1 3}$ \\
\hline & & Damage in 10th story \\
12th story & 0.0015 & 0.0013 & 0.0014 \\
11th story & 0.0010 & 0.0002 & 0.0003 \\
10th story & 0.0011 & 0.0003 & $\mathbf{0 . 0 2 2 4}$ \\
\hline
\end{tabular}

TABLE 2: Changes in the first AR model coefficient matrix for Substructure III (10\% damage).

\begin{tabular}{lccc}
\hline DOF & 3rd story & 2nd story & 1st story \\
\hline & \multicolumn{3}{c}{ Damage in 4th story } \\
3rd story & $\mathbf{0 . 0 2 2 9}$ & 0.0014 & 0.0006 \\
2nd story & 0.0001 & 0.0001 & 0.0005 \\
1st story & 0.0007 & 0.0008 & 0.0004 \\
\hline \multicolumn{4}{c}{ Damage in 3rd story } \\
3rd story & $\mathbf{0 . 0 2 2 2}$ & $\mathbf{0 . 0 2 1 1}$ \\
2nd story & $\mathbf{0 . 0 2 1 9}$ & $\mathbf{0 . 0 2 1 7}$ & 0.0006 \\
1st story & 0.0008 & 0.0016 & 0.0004 \\
\hline & & Damage in 2nd story \\
3rd story & 0.0000 & 0.0010 & 0.0005 \\
2nd story & 0.0003 & $\mathbf{0 . 0 2 2 4}$ & $\mathbf{0 . 0 2 1 5}$ \\
1st story & 0.0009 & $\mathbf{0 . 0 2 1 8}$ & $\mathbf{0 . 0 2 1 9}$ \\
\hline & \multicolumn{3}{c}{ Damage in 1st story } \\
3rd story & 0.0003 & 0.0001 & 0.0001 \\
2nd story & 0.0007 & 0.0004 & 0.0004 \\
1st story & 0.0002 & 0.0006 & $\mathbf{0 . 0 2 3 0}$ \\
\hline
\end{tabular}

listed in Table 1 for Substructure I in the case of 10\% damage occurring in 12th story, 11th story, and 10th story, respectively. The similar results for Substructure III are also listed in Table 2. The bold values in Tables 1 and 2 mean the large values compared to the rest of the elements of CFAR. It can be observed from Figure 4 and Tables 1 and 2 that the CFAR attains large values at the output DOFs next to the damaged location while the values in the rest of the positions are close to zero. Taking Substructure II as an example, the CFAR shows large value at the element $(8,8)$ when damage taking place in 9th story, and in the case of damage in 8th story, large values of CFAR appear at two diagonal elements $(8,8),(7,7)$ and two off-diagonal elements $(7,8),(8,7)$. This is in good agreement with the theoretical conclusion that the elements of CFAR corresponding to the output DOFs adjacent to the damaged location are proportional to the stiffness reduction in the structure, as shown in (23) (26).
TABle 3: Parameters of five-story building model.

\begin{tabular}{lcc}
\hline & $\begin{array}{c}\text { Column section } \\
h \times b \times l\left(\mathrm{~m}^{3}\right)\end{array}$ & $\begin{array}{c}\text { Theoretical stiffness } \\
(\mathrm{N} / \mathrm{m})\end{array}$ \\
\hline Column type 0 & $0.0025 \times 0.03 \times 0.24$ & $3.3908 \times 10^{3}$ \\
Column type 1 & $0.003 \times 0.014 \times 0.24$ & $2.7344 \times 10^{3}$ \\
Column type 2 & $0.003 \times 0.010 \times 0.24$ & $1.9531 \times 10^{3}$ \\
Column type 3 & $0.003 \times 0.006 \times 0.24$ & $1.1719 \times 10^{3}$ \\
\hline
\end{tabular}

Figure 5 shows a three-dimensional bar plot of the CFAR against output DOFs for Substructure II in the case of $10 \%$, $20 \%, 30 \%, 40 \%$, and $50 \%$ damage occurring in 7 th story, respectively. In addition, Figure 6 shows a plot of peak value of CFAR with five different damage intensities for all the three substructures. It is revealed from Figures 5 and 6 that there is a linear relationship between the peak values of CFAR and the damage intensities. This is in perfect accordance with the conclusion drawn from (23) (26), which were deduced by ignoring the higher order terms. Thus, it is clearly reasonable that a first-order approximation as derived earlier in (23) (26) is satisfactory to reveal the linear relationship between the peak values of CFAR and the damage intensities even in the case of a high intensity of damage. Therefore, one can draw a conclusion that the proposed substructural damage identification approach based on changes in the first AR model coefficient matrix (CFAR) is able to detect both location and severity of damage for shear structure in the simulation.

\section{Experimental Verification}

In this section, the measured data from a shake table experiment of a five-story frame structure was utilized to verify the performance of our proposed methodology.

4.1. Experimental Setup. The model structure is depicted in Figure 7(a). This experimental setup imitates a five-story shear frame building. The story mass was provided by the weight of aluminium floor slabs, bronze columns, and sensors, which is $4.360 \mathrm{~kg}$ for the 1st 4 th story and $3.544 \mathrm{~kg}$ for the 5th story. The interfloor stiffness was decided by the bronze plate springs which are shown in Figure 7(b). Young's modulus of bronze is $1.0 \times 10^{11} \mathrm{~N} / \mathrm{m}^{2}$. The cross section size and the theoretical stiffness in the weak-axis of each column are shown in Table 3 and Figure 7(c). The structure was initially healthy with all original columns intact (Column type 0 ). The damage was introduced in the experiment by replacing two original columns in the diagonal position of one story with two weaker ones. Three types of weaker columns were used in the experiment as shown in Figure 7(b) and Table 3.

The building model was placed on a shake table, as shown in Figure 7(a). The basement of the structure was set on bearings so that the structure could experience input motion. The force input to the structure was provided with an electrodynamic shaker. Accelerometers were installed on 

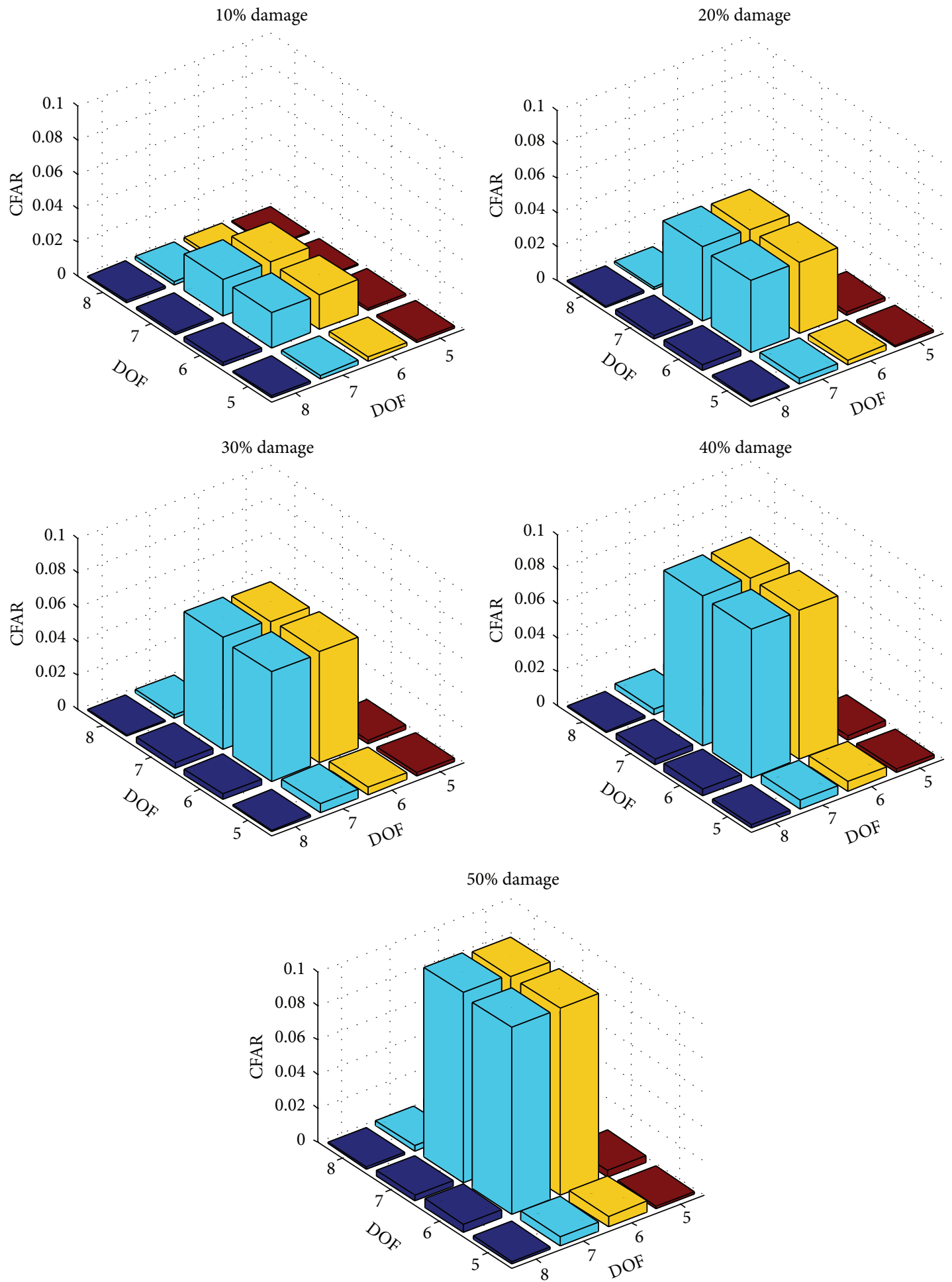

FIgURE 5: Changes in the first AR model coefficient matrix for Substructure II (damage in 7th story). 


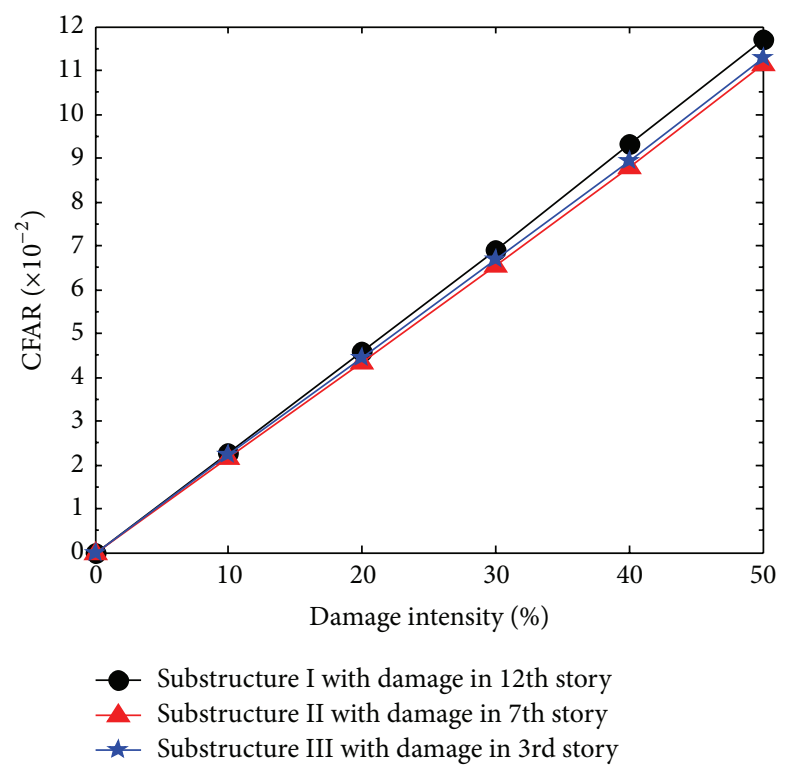

FIGURE 6: Linear relationship between CFAR and damage intensity.

each floor plate to measure the acceleration response, and one was installed on the basement to measure the input motion.

4.2. Procedure. A sinusoidal wave with frequencies sweeping from $1.0 \mathrm{~Hz}$ to $18.0 \mathrm{~Hz}$ was used as the input signal. The acceleration time histories were recorded for $180 \mathrm{~s}$ at a sampling frequency of $200 \mathrm{~Hz}$. Then they were decimated into $100 \mathrm{~Hz}$. A part of one typical acceleration time history measured is shown in Figure 8. An 8-order low-pass Butterworth filter with cut-frequency $40 \mathrm{~Hz}$ was applied to the measured responses in order to remove the effects of noise.

The five-story structure was firstly tested under the healthy state with all original columns. The force input to the structure was provided by the shaker to obtain the acceleration data of the healthy structure.

Then, two columns of one story were replaced by weaker columns to simulate the damage case. By replacing two original columns (Column type 0 ) in one story with three types of weaker ones (Column types 1, 2, and 3), the interfloor stiffness was reduced by around $10 \%, 20 \%$, and $30 \%$, respectively. The shaker provided excitation again in order to generate the acceleration data of the damaged structure, which were measured and recorded by the accelerometers. In total, data were collected for the undamaged state and fifteen (10\%, $20 \%$, and $30 \%$ damage in the 1st story, 2nd story, 3rd story, 4th story, and 5th story: $3 \times 5=15$ ) damaged cases.

The same procedures as the simulation cases were performed on the experimental measured data to detect both location and severity of damages in the test building model. First of all, following the substructure division rules described in Section 2, the whole structure was divided into 2 substructures, as shown in Figure 9.

According to (11), Substructure I was modeled as a 2-input 2-output ARMAX model. The ground acceleration $\left(\ddot{z}_{g}\right)$ and the acceleration of the 3 rd DOF relative to the ground $\left(\ddot{y}_{3}\right)$
TABLE 4: Changes in the first AR model coefficient matrix for Substructure I (10\% damage).

\begin{tabular}{lcc}
\hline DOF & 5th story & 4th story \\
\hline & \multicolumn{2}{c}{ Damage in 5th story } \\
5th story & $\mathbf{0 . 0 1 8 8}$ & $\mathbf{0 . 0 1 8 9}$ \\
4th story & $\mathbf{0 . 0 1 5 8}$ & $\mathbf{0 . 0 2 0 1}$ \\
\hline & \multicolumn{3}{c}{ Damage in 4th story } \\
5th story & 0.0027 & 0.0029 \\
4th story & 0.0010 & $\mathbf{0 . 0 1 6 2}$ \\
\hline
\end{tabular}

were used as the inputs, and the relative acceleration of the 4 th and 5th DOF to the 3rd DOF $\left(\ddot{y}_{4}^{r}\right.$ and $\ddot{y}_{5}^{r}$ ) was used as the outputs to construct the ARMAX model.

Similarly, Substructure II was also modeled as a 2-input 2-output ARMAX model. The ground acceleration $\left(\ddot{z}_{g}\right)$ and the acceleration of the 3 rd DOF relative to the ground $\left(\ddot{y}_{3}\right)$ were used as the inputs, and the relative acceleration of the 1st and 2nd DOF to the 3 rd DOF $\left(\ddot{y}_{1}^{r}\right.$ and $\ddot{y}_{2}^{r}$ ) was used as the outputs.

Next the changes in the first AR model coefficient matrix (CFAR) from the undamaged state to the damaged states were estimated to depict the damage existing in the experimental building model. As shown in Figure 10, the absolute values of CFAR are plotted against output DOFs in the form of a three-dimensional bar chart for Substructure II in the case of $10 \%$ damage occurring in 3rd story, 2nd story, and 1st story, respectively. Table 4 provides the absolute values of CFAR for Substructure I with $10 \%$ damage in 5 th story and 4 th story, respectively, in which the large values are bold. From Figure 10 and Table 4, it can be observed that large CFAR values are found at the output DOFs next to the damaged location, which is consistent with the previous theoretical derivation and the results of simulation verification. 

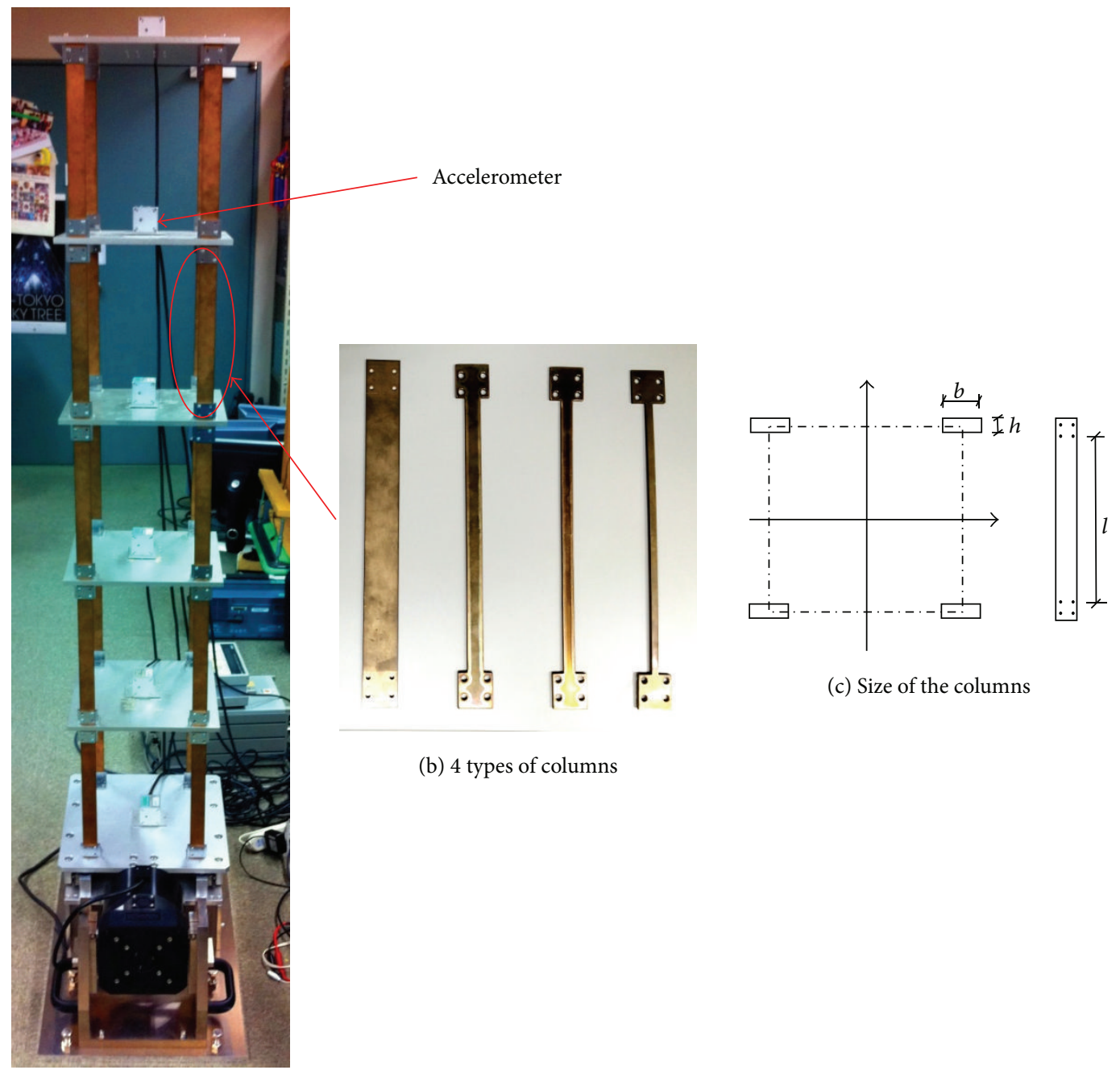

(c) Size of the columns

(b) 4 types of columns

(a) Building model

FIGURE 7: Experimental setup of five-story building model.

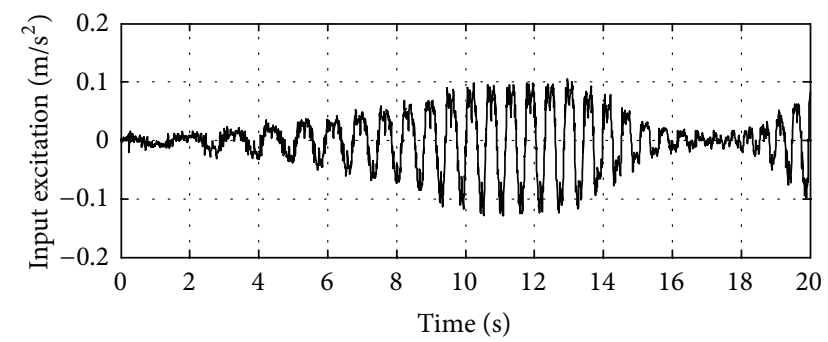

FIgURE 8: Input excitation.

Figure 11 presents the three-dimensional bar plot of the CFAR against output DOFs for Substructure II in the case of $10 \%, 20 \%$, and $30 \%$ damage occurring in 1st story, respectively. And Figure 12 shows a plot of peak value of CFAR with three different damage intensities for Substructures I and II. One can also notice from Figures 11 and 12 that the peak values of CFAR increase linearly with the damage intensities.
This is also in good agreement with the previous theoretical derivation and the results of simulation verification.

It is therefore clear that the proposed substructural damage identification approach based on changes in the first AR model coefficient matrix (CFAR) can still successfully locate and quantify the damage when applied to the experimental data. After experimentally verifying and confirming the feasibility and stability of the proposed methodology as discussed above, we may conclude that it can obtain satisfactory damage identification results in both simulation and laboratory experiment.

\section{Conclusions}

In this work, a substructural damage identification approach is presented to locate and quantify damages in a shear structure based on changes in the first AR model coefficient matrix (CFAR). At first, the mathematical derivation draws a conclusion that the elements of CFAR corresponding to the output DOFs adjacent to the damaged location are 


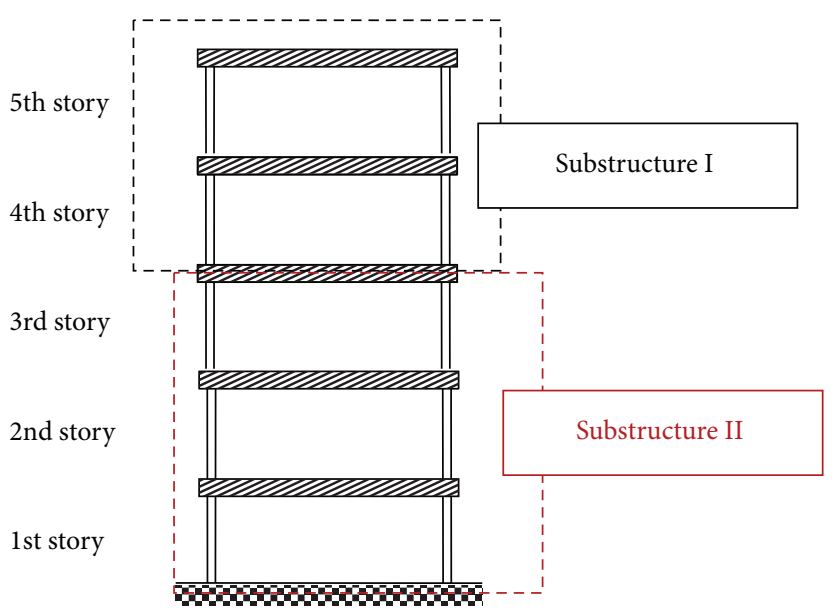

FIGURE 9: Structure division for 5-story experimental building model.
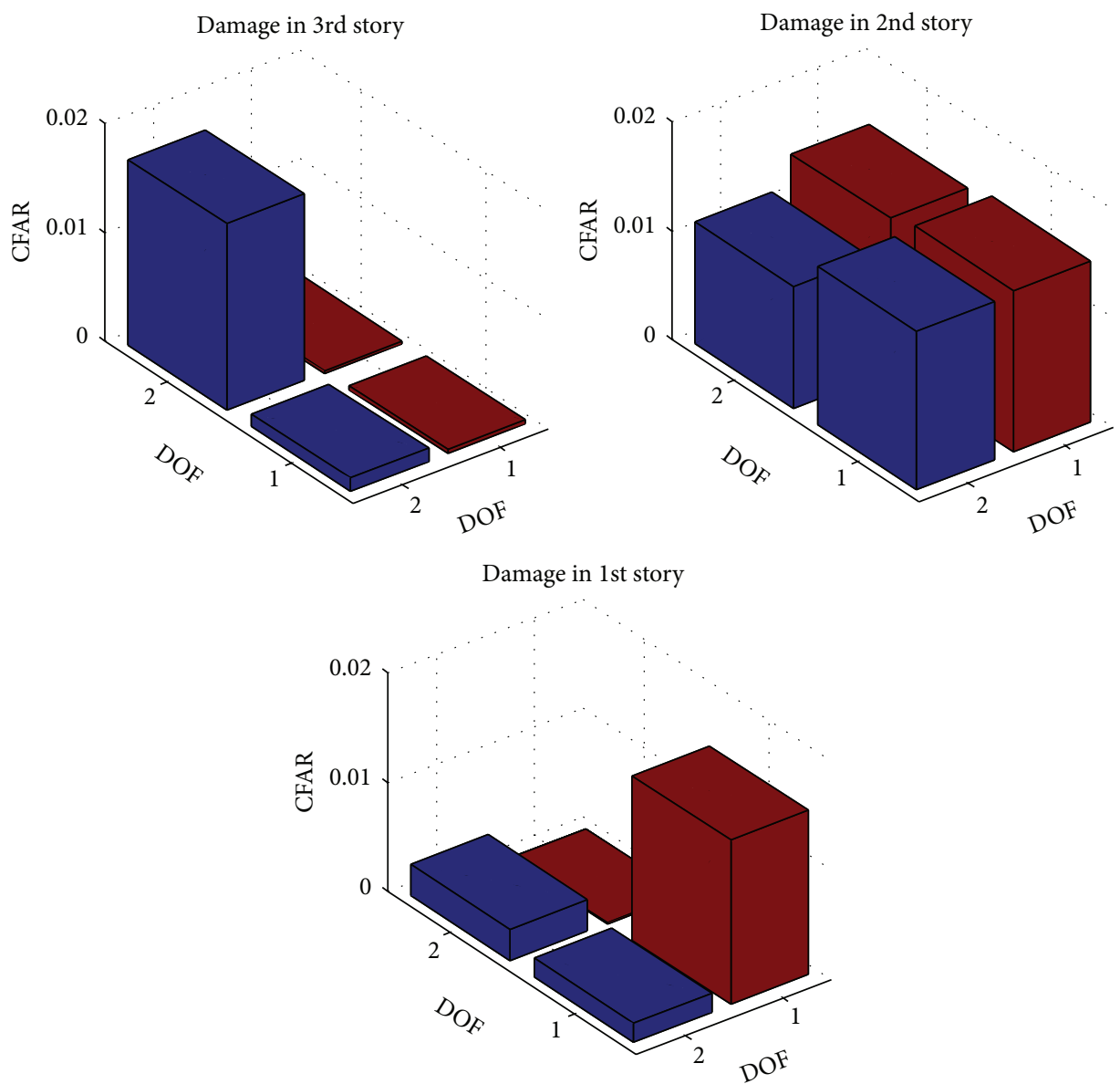

FIgURE 10: Changes in the first AR model coefficient matrix for Substructure II (10\% damage).

proportional to the stiffness reduction in the structure, indicating the damage location and severity. After that, a numerical simulation of a 12 -story shear building model and an experiment of a five-story metal building model have verified that the proposed approach can satisfactorily locate and quantify the damage in both simulation and laboratory experiment. As the structure is divided into substructures, which have a considerably smaller number of degrees of freedom (DOFs), thus only the structural responses related to the substructure being detected are required in the substructural damage identification procedure, and it is not necessary to monitor the responses of all DOFs of the structure 

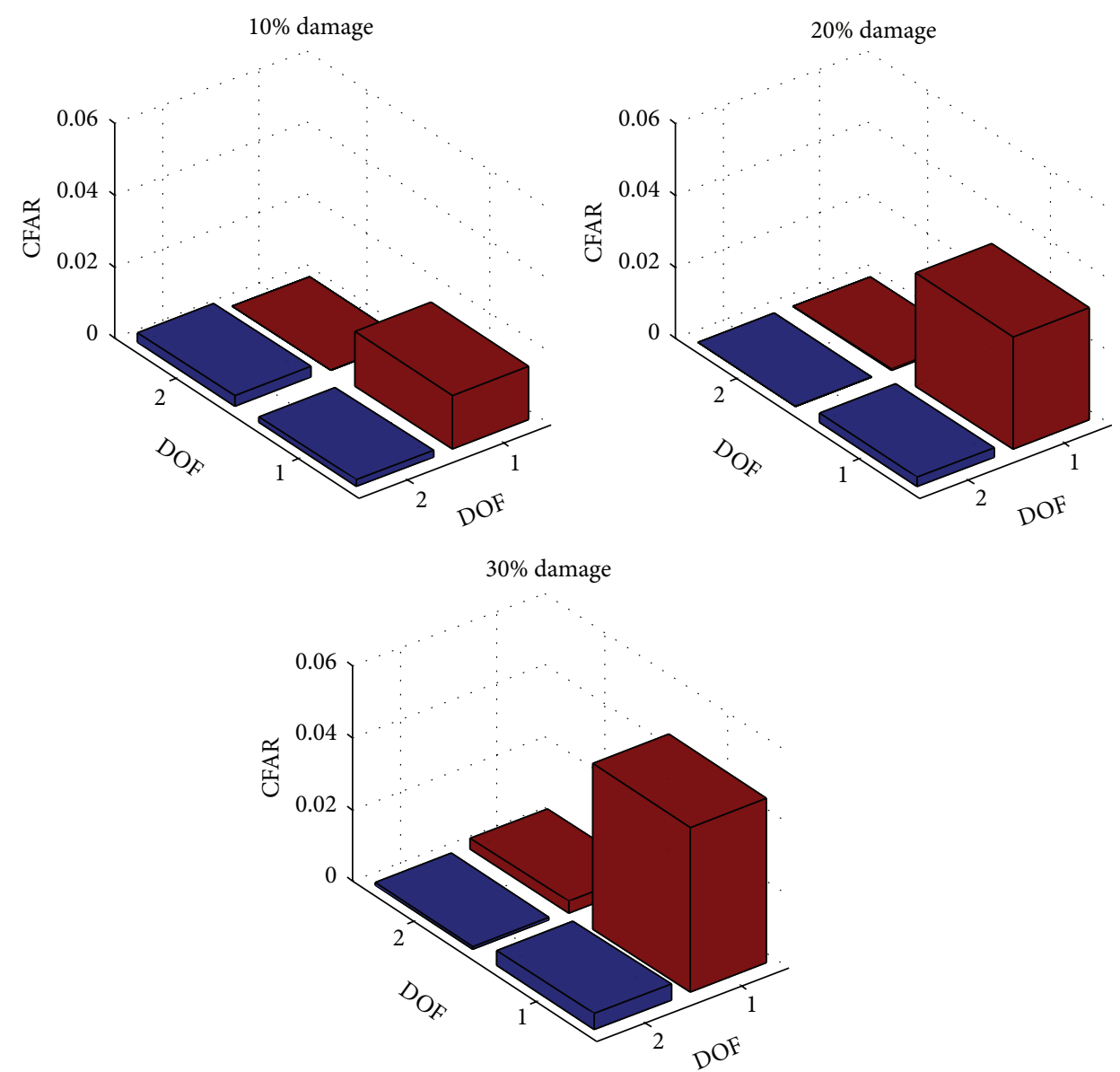

FIGURE 11: Changes in the first AR model coefficient matrix for Substructure II (damage in 1st story).

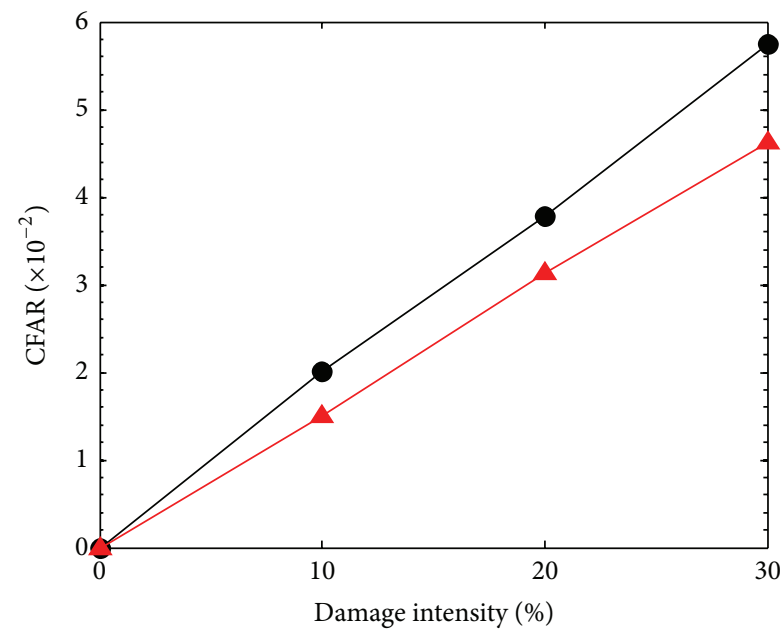

Substructure I with damage in 5th story

Substructure II with damage in 1st story

FIGURE 12: Linear relationship between CFAR and damage intensity.

simultaneously, greatly reducing the computation time and cost of SHM systems. It may also be noted that the evaluation of the AR model coefficient matrix involves only very easy and simple computation, without having to take long time to identify the mass, stiffness, and damping matrices of the structure. This means that the proposed approach is easy, efficient, and stable for local substructural damage identification of shear structures. Moreover, as the damage identification process can be independently conducted on each substructure, this method is promising for application in a parallel and distributed damage identification system (e.g., using wireless mobile sensor network to perform SHM). However, in this study the laboratory experimental data is still from a simplified structure subject to linear damages in a controlled operational and environmental condition. In the further study, more realistic conditions involving operational and environmental variations in the data should be taken into consideration, in order to challenge the damage identification and to test the robustness of the algorithm.

\section{Conflict of Interests}

The authors declare that there is no conflict of interests regarding the publication of this paper. 


\section{Acknowledgment}

This work was supported in part by a grant for the project "Implementation of Shelter Guidance System for Commuters Who Are Unable to Return Home Based on Structural Health Monitoring of Tall Buildings after Large-Scale Earthquake" (FY2013-2016, PI: A. Mita) from the Japan Science and Technology Agency.

\section{References}

[1] A. Mita, Structural Dynamics for Health Monitoring, Sankeisha, Nagoya, Japan, 2003.

[2] K. F. Alvin, A. N. Robertson, G. W. Reich, and K. C. Park, "Structural system identification: from reality to models," Computers \& Structures, vol. 81, no. 12, pp. 1149-1176, 2003.

[3] L. Ljung, System Identification: Theory for the User, Prentice Hall, New York, NY, USA, 1987.

[4] P. Cawley and R. D. Adams, "The location of defects in structures from measurements of natural frequencies," The Journal of Strain Analysis for Engineering Design, vol. 14, no. 2, pp. 49-57, 1979.

[5] S. Doebling and C. Farrar, "Using statistical analysis to enhance modal-based damage identification," in Proceedings of the DAMAS Conference, Sheffield, UK, 1997.

[6] S. W. Doebling, C. R. Farrar, M. B. Prime, and D. W. Shevitz, Damage Identification and Health Monitoring of Structural and Mechanical Systems from Changes in Their Vibration Characteristics: A Literature Review, Los Alamos National Laboratory, Los Alamos, NM, USA, 1996.

[7] S. D. Fassois and J. S. Sakellariou, "Time-series methods for fault detection and identification in vibrating structures," Philosophical Transactions of the Royal Society A, vol. 365, no. 1851, pp. 411-448, 2007.

[8] H. Sohn and C. R. Farrar, "Damage diagnosis using time series analysis of vibration signals," Smart Materials and Structures, vol. 10, no. 3, pp. 446-451, 2001.

[9] K. K. Nair, A. S. Kiremidjian, and K. H. Law, "Time series-based damage detection and localization algorithm with application to the ASCE benchmark structure," Journal of Sound and Vibration, vol. 291, no. 1-2, pp. 349-368, 2006.

[10] P. Omenzetter and J. M. W. Brownjohn, "Application of time series analysis for bridge monitoring," Smart Materials and Structures, vol. 15, no. 1, pp. 129-138, 2006.

[11] O. R. de Lautour and P. Omenzetter, "Detection of seismic damage in buildings using time series analysis and pattern recognition," in Proceedings of the 4th World Conference on Structural Control and Monitoring, San Diego, Calif, USA, July 2006.

[12] P. J. Fanning and E. P. Carden, "Auto-regressive and statistical process control techniques applied to damage indication in telecommunication masts," Key Engineering Materials, vol. 204205, pp. 251-260, 2001.

[13] S. G. Mattson and S. M. Pandit, "Statistical moments of autoregressive model residuals for damage localisation," Mechanical Systems and Signal Processing, vol. 20, no. 3, pp. 627-645, 2006.

[14] C. G. Koh, L. M. See, and T. Balendra, "Estimation of structural parameters in time domain. A substructure approach," Earthquake Engineering and Structural Dynamics, vol. 20, no. 8, pp. 787-801, 1991.
[15] C.-B. Yun and H.-J. Lee, "Substructural identification for damage estimation of structures," Structural Safety, vol. 19, no. 1, pp. 121-140, 1997.

[16] K. C. Park, G. W. Reich, and K. F. Alvin, "Structural damage detection using localized flexibilities," Journal of Intelligent Material Systems and Structures, vol. 9, no. 11, pp. 911-919, 1998.

[17] K. F. Tee, C. G. Koh, and S. T. Quek, "Substructural firstand second-order model identification for structural damage assessment," Earthquake Engineering and Structural Dynamics, vol. 34, no. 15, pp. 1755-1775, 2005.

[18] C. G. Koh and K. Shankar, "Substructural identification method without interface measurement," Journal of Engineering Mechanics, vol. 129, no. 7, pp. 769-776, 2003.

[19] Z. Xing and A. Mita, "A substructure approach to local damage detection of shear structure," Structural Control and Health Monitoring, vol. 19, no. 2, pp. 309-318, 2012.

[20] J. Hou, Ł. Jankowski, and J. Ou, "Experimental study of the substructure isolation method for local health monitoring," Structural Control and Health Monitoring, vol. 19, no. 4, pp. 491510, 2012.

[21] M. Kuwabara, S. Yoshitomi, and I. Takewaki, "A new approach to system identification and damage detection of high-rise buildings," Structural Control and Health Monitoring, vol. 20, no. 5, pp. 703-727, 2013.

[22] D. Zhang, H. Li, and Y. Bao, "Substructure parameter estimation for shear structures with limited measurements and unknown structural mass," in Sensors and Smart Structures Technologies for Civil, Mechanical, and Aerospace Systems, vol. 9061 of Proceedings of SPIE, San Diego, Calif, USA, 2014.

[23] E. T. Lee and H. C. Eun, "A model-based substructuring method for local damage detection of structure," Shock and Vibration, vol. 2014, Article ID 390769, 11 pages, 2014.

[24] C. G. Koh, B. Hong, and C. Y. Liaw, "Substructural and progressive structural identification methods," Engineering Structures, vol. 25, no. 12, pp. 1551-1563, 2003.

[25] Y. Lu and F. Gao, "A novel time-domain auto-regressive model for structural damage diagnosis," Journal of Sound and Vibration, vol. 283, no. 3-5, pp. 1031-1049, 2005. 

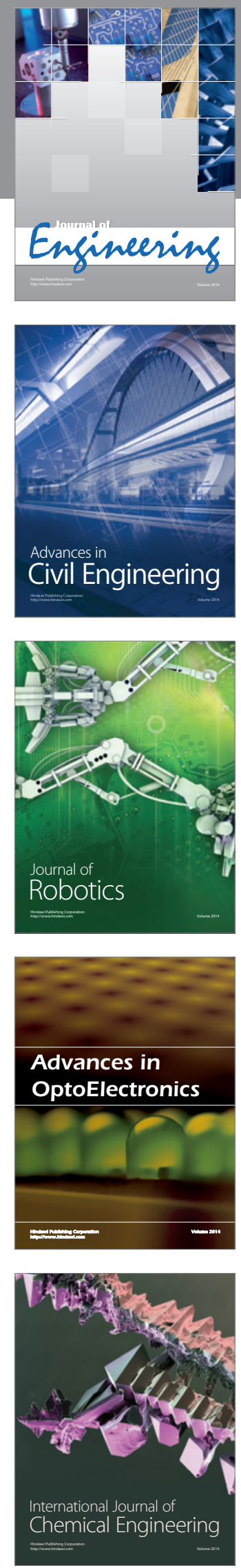

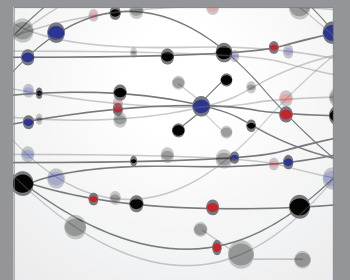

The Scientific World Journal
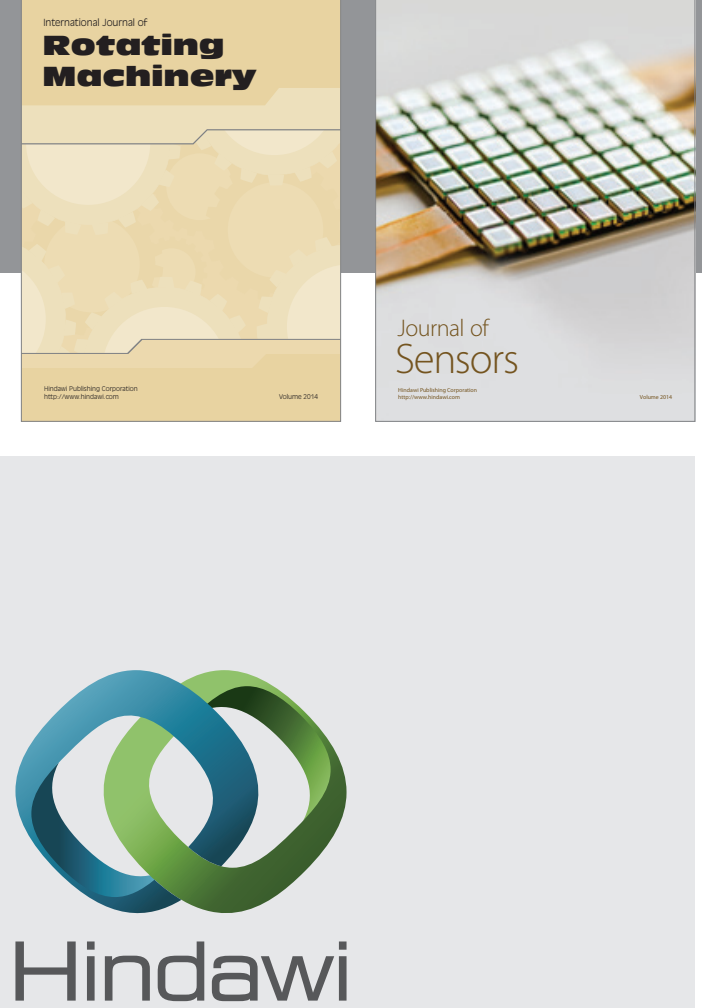

Submit your manuscripts at http://www.hindawi.com
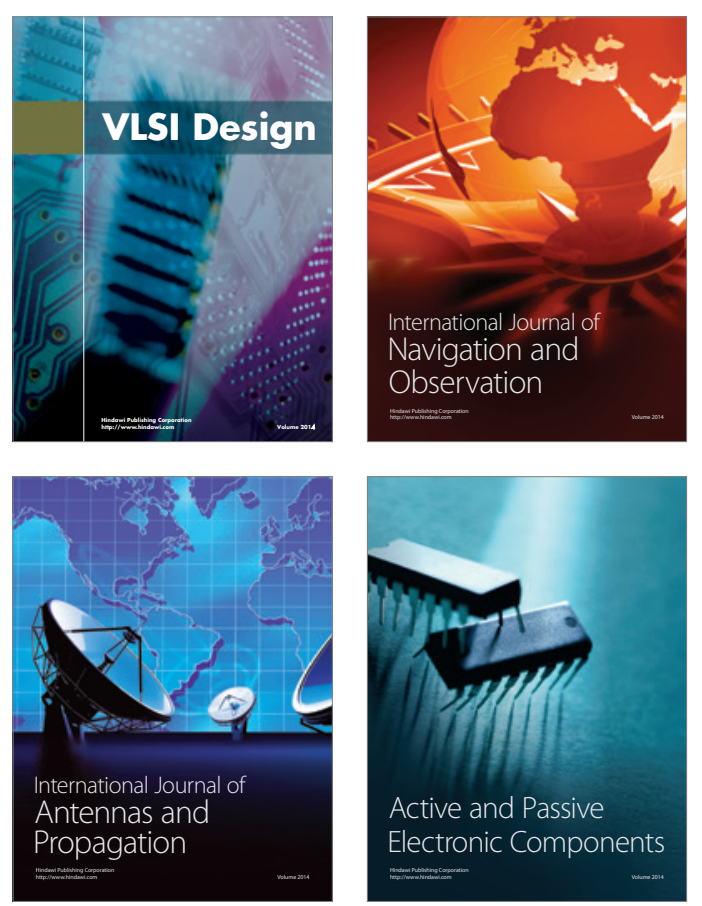
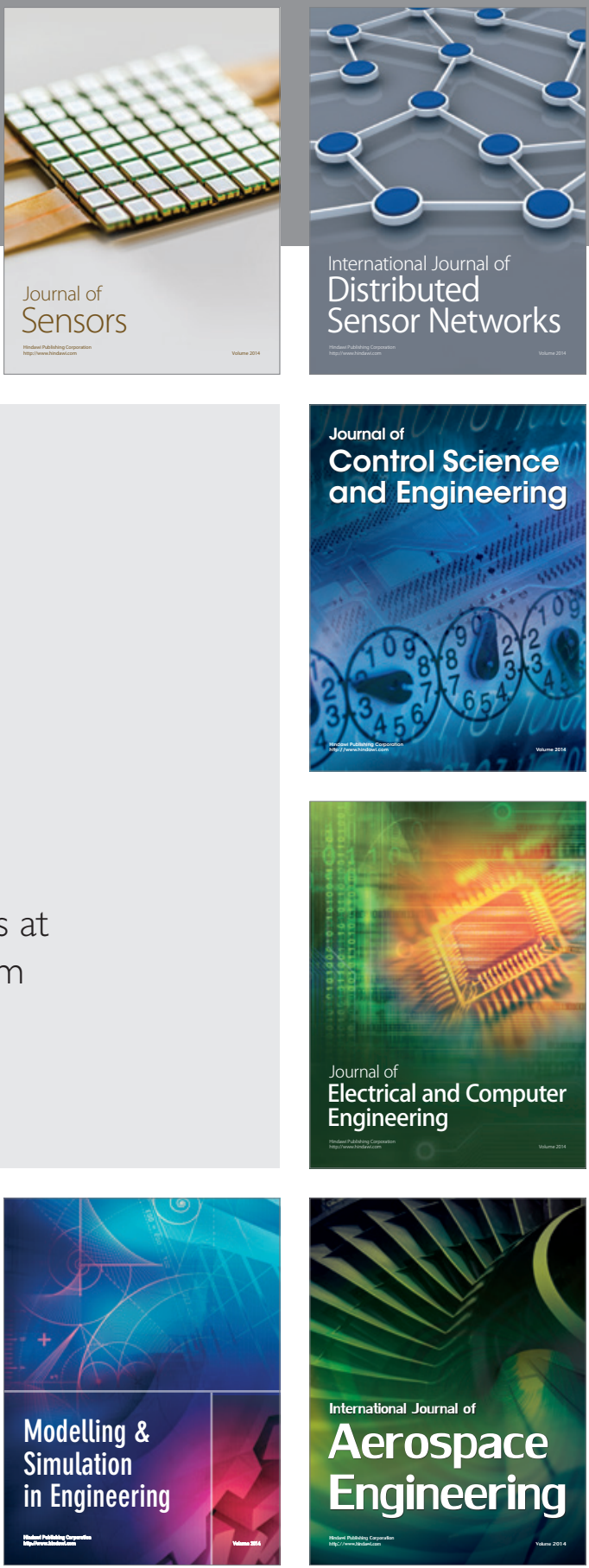

Journal of

Control Science

and Engineering
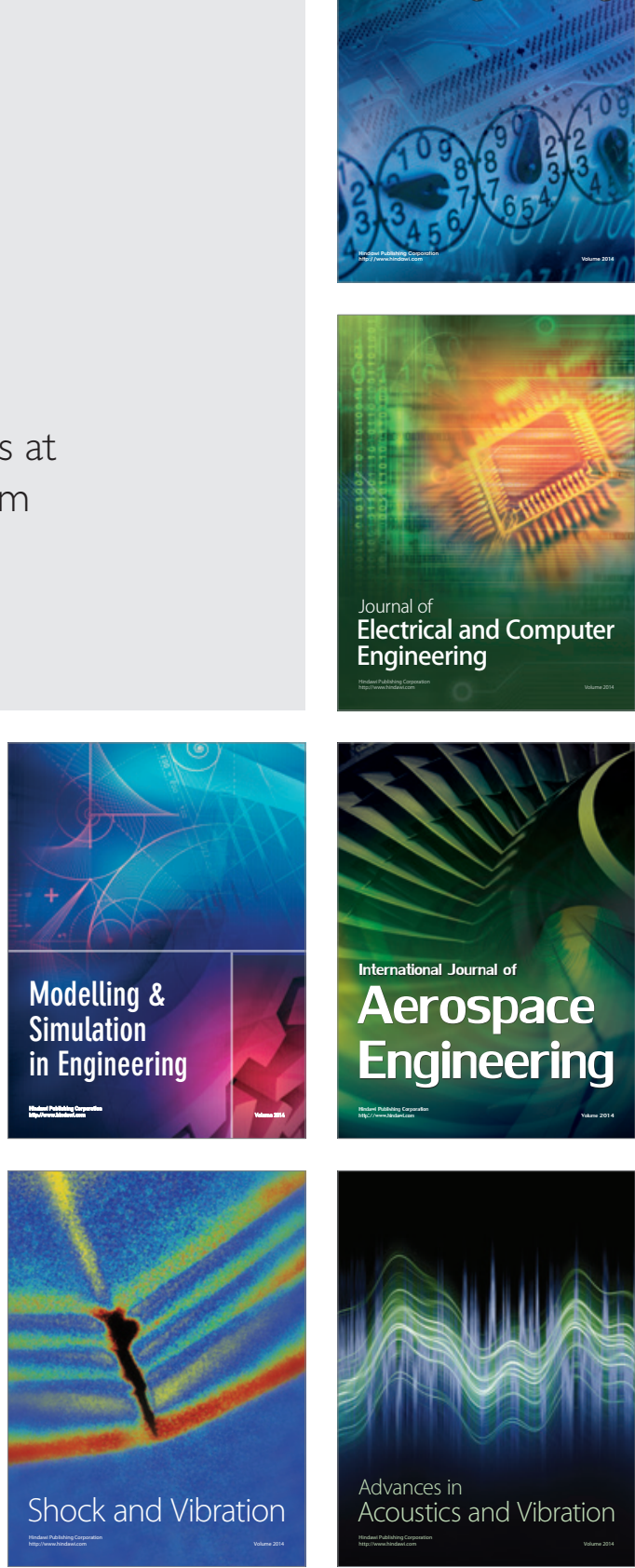